\title{
A Study of Charge Propagation and Quantification of Noise Within the SuperCDMS Detector
}

by

Thomas B. Binder

\begin{abstract}
A Thesis Submitted in Partial Fulfillment
Of the Requirements for the

University Honors Program
\end{abstract}

Department of Physics

The University of South Dakota

May 2016 
The members of the Honors Thesis Committee appointed to examine the thesis of Thomas B. Binder

find it satisfactory and recommend that it be accepted.

\begin{tabular}{c} 
Dr. Joel Sander \\
Assistant Professor of Physics \\
Director of the Committee \\
Associate Professor of Physics \\
Dr. Yongchen Sun \\
\hline
\end{tabular}

Dr. Jing Liu

Chair/Professor of Mathematical Sciences 


\author{
ABSTRACT \\ Thomas B. Binder \\ Director: Dr. Joel Sander, Ph. D.
}

This paper discuses two projects that were designed around the SuperCDMS experiment. The first is an attempt to understand the SuperCDMS simulation that was designed in the Matlab environment. The tests that were conducted were designed to study how electron and hole propagation is effected by the initial event location and the charge bias within the detector. The second experiment was an attempt to systematically quantify the level of noise produced within the SuperCDMS detectors by the dilution refrigerator. The results show only initial findings and no conclusive results were obtained.

KEYWORDS: Dark Matter, Propagation, Simulation, SuperCDMS, Dilution Refrigerator, Background Noise, MySQL, Matlab 


\section{Contents}

1 Introduction $\quad \mathbf{7}$

1.1 What is Dark matter . . . . . . . . . . . . . . 7

1.2 Leading Candidates for Dark Matter . . . . . . . . . . . . 8

1.3 What is SuperCDMS and Why is it Important . . . . . . . . . . 10

1.3.1 The SuperCDMS Experiment . . . . . . . . . . . . 10

1.3.2 The SuperCDMS Detector . . . . . . . . . . . . 13

1.3.3 The Importance of Background . . . . . . . . . . . . . . . 15

2 Simulating Propagation of Charged Particles in the SuperCDMS Detector 20

2.1 What and Why ................... 20

2.2 The Simulation . . . . . . . . . . . . . . . . . 21

2.2 .1 Testing the Simulation . . . . . . . . . . . . . . 23

2.2 .2 Unusual Propagation Features _. . . . . . . . . . 26

2.2.3 The Effect of the Electric Field on Charge Propagation . 28

2.3 Chapter Summary . . . . . . . . . . . . . . . . . . . 31

3 A Systematic Quantification of SuperCDMS Noise 33

3.1 What and Why . . . . . . . . . . . . . 33

3.2 Fridge Parameter- Detector Noise Study _ . . . . . . . . . 36

3.2 .1 Setup . . . . . . . . . . . . . . 36

3.2 .2 Implementing the Program . . . . . . . . . . . . . . 40

3.2 .3 Analysis . . . . . . . . . . . . . . . . . . . . . . 43

3.3 Chapter Summary . . . . . . . . . . . . . . . . . . . . 45

4 Outlook 47

4.1 Simulation Outlook . . . . . . . . . . . . . . . 47

4.2 Analysis Outlook . . . . . . . . . . . . . . . . . . . . 49

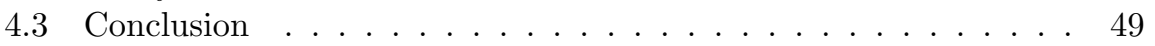




\section{List of Figures}

1.1 A comparison between the expected (A) and observed (B) rotational velocities of stars in a spiral galaxy. The observed velocity does not follow the same trend, which means something must be causing this discrepancy. . . . . . . . . . . . . . . .

1.2 This graph shows what is known as the "WIMP Miracle", it details how cross-section and density interact. The WIMP miracle is an interesting phenomena that uses supersymmetric predictions for the initial amount of WIMPs and the natural decay over time to predict how much should currently be in our universe. This predicted amount of WIMPs almost perfectly matches the amount that we have been able to see experimentally from indirect detection. $[8] \ldots \ldots \ldots \ldots \ldots \ldots$. . . . . . . . . . .

1.3 A model of many leading collaborations current and predicted detection ranges. The solid lines represent current detection curves, the dashed lines represent predicted detection ranges, and the bubbles show ranges where potential WIMP events have been detected. The dashed line at the bottom of the graph represents a natural barrier for the detection of WIMPs, as it's when neutrinos can cause interactions. As one can see SuperCDMS is the leader in low mass detection. $[8] \ldots \ldots$. . . . . . . . .

1.4 A highly simplified schematic view of only one component of the SuperCDMS fridge. As one can see the fridge is extremely complex 13

1.5 An image of an iZIP detector within its copper case. This detector has a $76 \mathrm{~mm}$ diameter and is $25 \mathrm{~mm}$ high. At the surface of the detector, the phonon sensors are clearly visible. . . . . . . . .

1.6 An schematic overview of a SuperCDMS detector. As can be seen the detector contains a Q-outer and Q-inner electrode. . . . . . .

1.7 An schematic overview of the phonon sensors on one side of a SuperCDMS detector. As can be seen the detector contains 3 inner phonon channels and one outer. The reason behind the larger amount of inner channels is that the primarily targeted events are ones that occur within the bulk of the inner electrode. 
1.8 A modeled image of how an event occurring near the surface of an iZIP detector would react. As one can see that when an event occurs near the surface both the electrons and holes travel to the same surface side due to the electric field. This configuration helps discriminate surface events as they can lead to false nuclear recoil events. . . . . . . . . . . . . . . .

1.9 An image of channel from the SuperCDMS experiment. As can be seen in this image there is a large amount of background within the center channel on the bottom graph, which can be determined by the large fluctuation. Note that the large peak seen on both of these graphs is an event. . . . . . . . . . . . . . .

2.1 A simulated image of an event occurring in an iZIP detector. The event occurred at a radial position of $.015 \mathrm{~m}$ and at a height of about $1 \mathrm{~cm}$ above the bottom of the detector. The vertical line at a radial position of $0.032 \mathrm{~m}$ denotes the boundary between the portion of the detector covered by the inner charge electrode and that of the outer electrode. Holes propagate upward following the electric field and electrons have a smaller effective mass and spread out more as the propagate down towards the bottom of the detector. The surface electric field described in section 1.3.2 causes the arching feature as they approach the top and bottom of detectors. Here the holes are represented by the lighter color traveling towards the top of the detector and the electrons are shown in the dark color traveling towards the bottom. . . . . . .

2.2 A simulated image of an event occurring in the bulk of an iZip detector. The simulated image is similar to that in shown in figure 2.1 , except its initial event position was changed to $(.03, .02)$. Here the holes are represented by the lighter color traveling towards the top of the detector and the electrons are shown in the dark color traveling towards the bottom. It is interesting to note that as the electrons hit the boundary between the inner and outer charge electrode their spread is effected. . . . . . . . . . . .

2.3 A graph detailing the simulated spread difference based on the event location in the detector. Electrons are shown by the dark color and have greater spread. The holes are shown as the lighter color and contain a lesser spread. . . . . . . . . . . . .

2.4 A simulated image of a surface iZIP event. This image is zoomed into the center of the detector. The electrons are shown moving to the left and the holes are shown moving to the right. Note: the surface of the detector is located at $.0254 \mathrm{~m}$, the extra space above this point is outside the detector. . . . . . . . 26 
2.5 An image of two simulated events occurring within the outer electrode. As one can see the charged particles propagate towards the side of the detector which is where the grounded copper shield is located. The dark color represents the electrons and the holes are represented by the lighter color. . . . . . . . . . . . .

2.6 A plot that shows how voltage effects propagation. As usual the dark color represents electrons and the lighter color shows holes. As one can see the holes have a linear tendency when voltage is being varied. However holes do not contain this tendency and seem to exponentially increase in spread size as the field decreases. 29

2.7 A zoomed in image of a simulated event occurring in at the initial location $(.01, .01, .01) \mathrm{m}$ in a standard CDMS detector. Here the holes are represented by the lighter color traveling towards the top of the detector and the electrons are shown in the dark color traveling towards the bottom. This low voltage $(.02 \mathrm{~V} /$ $\mathrm{cm}$ of detector thickness) test shows the spread is very large and relatively messy. . . . . . . . . . . . . . .

2.8 A zoomed in image of a simulated event occurring in at the initial location $(.01, .01, .01) \mathrm{m}$ in a standard CDMS detector. Here the holes are represented by the lighter color traveling towards the top of the detector and the electrons are shown in the dark color traveling towards the bottom. This high voltage $(50 \mathrm{~V} / \mathrm{cm}$ of detector thickness) test shows the spread is very small and clean.

2.9 A graph from the PDG that shows the absorption length at different photon energy levels. As one can see elements like Si that contain a similar density as Ge are shown in this graph. This graph can be used to see whether or not a normal event within a SuperCDMS detector could travel far enough to reach the sensors at the surfaces. . . . . . . . . . . . . . . . . . . .

3.1 A live schematic snapshot of the SuperCDMS helium chamber. This image is just one of the many components that make up the SuperCDMS fridge. As one can see the fridge is very complex which greatly increases the chance of background noise being introduced to the experiment. Therefore it is crucial to try and minimize this background noise as much as possible. [8] . . . .

3.2 A live schematic snapshot of the SuperCDMS icebox. Like figure 3.1 , this image is just one of the many components that make up the SuperCDMS fridge. As one can see this fridge is very complex which increases the chance of background noise being introduced to the experiment. Therefore it is crucial to try and minimize this background noise as much as possible. [8] . . . . . 
3.3 This graph is a screen shot of one detector temperature variable from the SuperCDMS fridge. This is important to examine because it contains a well known pattern that could be used to compare with my project's test results. As on can see there are peaks in the temperature every three hours. This is a result from flashing the detectors. Once this is complete the detectors are cooled again to around $40 \mathrm{mK} . \ldots \ldots \ldots$. . . . . . .

3.4 This graph shows how the temperature of the Outer Vacuum Can (OVC) varies over time. This plot was used initially to determine if the program was working correctly. When compared with the values from the online SuperCDMS fridge historical plotter it was clear that this program was indeed behaving correctly. This was confirmed by matching series numbers with their given time stamps. If the two series numbers and the time stamps were both the same we knew that the two were properly aligned. . . . . . .

3.5 Each point on this plot represents a minutes worth of fridge data that has been averaged. SuperCDMS takes a snapshot of their data every 3 seconds which makes this amount that was dealt with in this experiment very large and complex. It is also important to note that it looks like there are two populations or a correlation between the fridge variables. This may be an important result but it could also be coincidental. Therefore, further testing was required to discover if this was anything of value. . . . . . . . .

3.6 A plot that shows the standard deviation of the Ovc can temperature compared to volts. This series of data had all of the outliers removed that were not within the set parameter of $3 \sigma$. This kind of data cut is important to apply because it allows us to examine the bulk of data rather than the outliers. Each point on this plot represents a minutes worth of fridge data that has

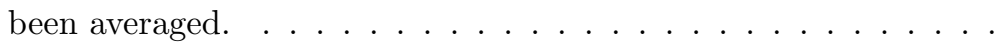

3.7 This plot shows the average correlation coefficient between detector noise and each of the 273 fridge variables. The $\mathrm{x}$-axis gives the average correlation coefficients for series 1406 . The y-axis gives the average correlation coefficients for series 1407A. Each point represents a different fridge variable's correlation coefficient averaged over all detector noise levels. As on can see the bulk of the averaged correlation coefficients are located near the point of zero correlation $(0,0)$ which is to be expected. However some coefficients have values that may be significantly far from zero. A large correlation coefficient might indicate a possible correlation between a fridge variable and detector noise level. I chose a threshold (|averaged correlation coefficient $\mid>0.75$ ) for identifying coefficients with higher levels of correlation (indicated by the blue boxes) and used these to search for potential culprits. The circled point is the value $\mathrm{I} \ldots \ldots \ldots \ldots \ldots$ 
4.1 This image is a few frames from the rotating plotter that I edited for this simulation. As one can see this can be very useful because the user can view what the propagation looks like at different angles and at different times. As usual the holes are represented by the lighter color traveling up and the electrons are the darker color traveling downwards. The times given in each frame is the time since the particle interaction in the detector. . . . . . . . . 48 


\section{Chapter 1}

\section{Introduction}

\subsection{What is Dark matter}

During the beginning of human history there was a belief that only what we could see is what is real, but that all changed when the Greek philosophers imagined that there were tiny particles making up everything around us. Since that moment in history, humans have been on a quest to discover the invisible particles that make up our world. Now fast forwarding to modern day, we find ourselves on the same quest as our predecessors. The primary focus today, however, is on what is known as dark matter, which is a mysterious substance that makes up nearly 27 percent of the universe. [5] Now 27 percent may seem like a small number, but when it's compared to how much normal matter (atoms,neutrinos, etc.) it is actually a huge portion of our universe. In fact normal matter only makes up only 5 percent of our universe. [5] Therefore, it is important to understand this mysterious substance known as the dark matter.

To better understand this idea of dark matter, first we need to know where this idea came from and how we actually know it's real. The whole idea of dark matter began with a famous observation done by Vera Rubin in the 1970's, where she discovered that there was a discrepancy between the predicted rotational velocity of our galaxy and that of the observed rotational velocity. [7] According to classical mechanics we know that the rotational velocity should fall off at an inverse rate, which can be seen by the following derivation.

$$
\text { Force }_{\text {gravity }}=\frac{G M m}{r^{2}}
$$

Here our $\mathrm{G}$ is the gravitational constant, $\mathrm{M}$ is the mass of the earth (or the fixed object of your calculation), $\mathrm{m}$ is the mass of another object, and $\mathrm{r}$ is the distance between the two masses. Therefore Newton's Second Law for circular motion tells us

$$
\text { Force }=\text { mass } * \frac{m v^{2}}{r}
$$




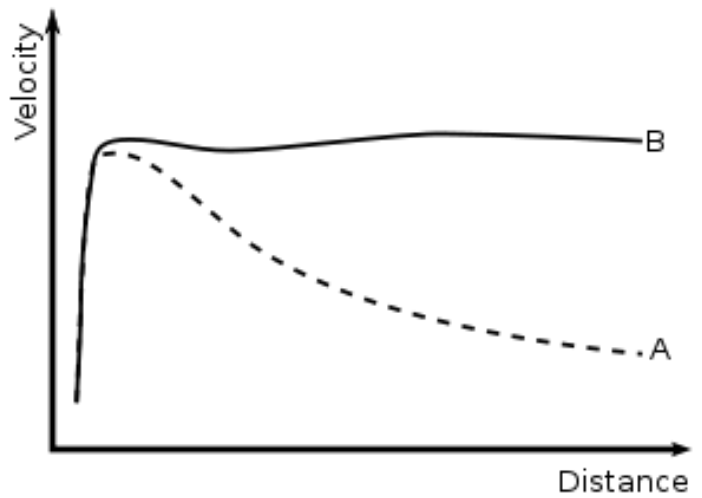

Figure 1.1: A comparison between the expected (A) and observed (B) rotational velocities of stars in a spiral galaxy. The observed velocity does not follow the same trend, which means something must be causing this discrepancy.

Now equating the force equations, we get

$$
\frac{G M m}{r^{2}}=\frac{m v^{2}}{r}
$$

Solving for the rotational velocity, we find

$$
v=\sqrt[2]{G M / r}
$$

As one can see by this formula we would expect for the rotational velocity of stars in the galaxy to drop off inversely as the square root of the radius increases. However, when Vera Rubin made her observations she found that galaxies did not follow this trend; instead the galaxies' velocity tended to level off and even increase as the distance from the center increased as shown in 1.1. In order to solve this mystery, Rubin postulated that there was a some sort of nonvisible matter was greatly increasing the galaxy mass. Thud the modern idea of dark matter was formed. There have been many more observations ranging from the bullet cluster to gravitational lensing [4], that have helped confirm the existence of dark matter. In the interest of space I will not be detailing these here. Therefore it is reasonable to say that tstrong evidence for the existence of dark matter. The only question is: what makes up dark matter?

\subsection{Leading Candidates for Dark Matter}

Now that we know a little about the reasons why dark matter likely exists, we can discuss what types of particles makes up this mysterious substance. There are currently three major theories: MACHOs, Axions and WIMPs. The first, which are known as a MACHOs (Massive Astrophysical Compact Halo Objects), 
are normal baryonic particles. The most common theory is that these MACHOs consist of hot white dwarfs and black holes. [4] They are believed to give off very little radiation, which make them very hard to detect. The primary reason MACHOs are considered a candidate, is that they explain the clumping, halo effect that we see on the outside of galaxies. [5] However, MACHOs have fallen out of favor in the scientific community due to results from the 2007 run of the EROS-2 microlensing survey. [2] These results suggested that our galaxy's halo would not be composed primarily of MACHOs with a mass in the range of M/M is less than 0.6107 and greater than 15. [4] Due to this result, MACHOs are expected to be no more than a small fraction of the dark matter.

The second primary candidates is what is known as axions. These particles were conceived by R.D. Peccei and Quinn, in order to solve what is known as the strong CP problem [6]. In short the strong CP problem is that Quantum Chromodynamics (QCD) does not violate the charge parity symmetry. In theory a very strong interaction like $\mathrm{QCD}$ should violate the $\mathrm{CP}$ parity because the QCD's Lagrangian contains a variable that is not consistent with CP. However, when observed experimentally, QCD actually preserves CP. [6] This issue lead to the formation of a scalar-particle, known as an axion, that could potentially solve this issue. An axion would contain no electric charge, would have a very low mass would have a extremely low cross-section, much like WIMPs, with an energy in the order of $10^{-6}$ to $1 \frac{e V}{c^{2}}$ [6]. However, axions do leave many unanswered questions if they were the main particle that made up dark matter. One of these questions is that it does not correctly predict the issue with $\mathrm{x}$ rays. Xrays should to have a black-body spectrum that peaks at keV energies, however the spectrum show more of a form that does not have keV peaks. [1] In addition there is the issue with non-relic dark matter. Since in theory axions are still produced they don't quite match up with the predicted amount of dark matter left from the early universe, further making them a non-perfect candidate. Despite this flaw, these properties would also make the axion a good candidate for dark matter.

The third proposed particle is the the WIMP. A WIMP is a weakly interacting massive particle that has an expected mass of about $1 \mathrm{GeV}$ to $10 \mathrm{TeV}$. [8] In addition, it is believed that WIMPs have an extremely small interaction rate with normal matter, which makes them very difficult to detect. WIMPs are the most likely candidate for dark matter because they solve multiple issues in the world of physics. One of the major issues that is solved is a problem with the theory known as Supersymmetry. Supersymmetry is a theory based off of the Standard Model in physics. Essentially, this theory states that there is a symmetry between fermionic and bosionic fields. The reason behind this is because fermions contain a half spin number (like $1 / 2,3 / 2,5 / 2$ ) and a boson has a whole number spin (like 1,2,3) In addition, this theory introduces R-parity, which states that all Standard Model particles are equal to +1 and all Supersymmetry partner particles are equal to -1 . Therefore we know through particle physics that neither of these will decay when R-parity is conserved. [4] WIMPs naturally occur in supersymmetric theories and are a great candidate for dark matter. Another major issue that is solved by WIMP dark matter is 
the thermal physics after expansion. According to big bang nucleosynthesis, shortly after inflation was complete, a massive particle would be in total thermal equilibrium. Due to this, when the universe's expansion begins to decrease, the annihilation of these massive particles with one another stops. Therefore it is fair to assume that these massive particles will have a very small interaction cross-section. This is consistent with what the proposed WIMP cross sectional energy of $3 \times 10^{-26} \mathrm{~cm}^{3} \mathrm{~s}^{-1}$. Therefore the WIMP is the most favored candidate for dark matter because its existence would solve multiple major problems in physics.

\subsection{What is SuperCDMS and Why is it Impor- tant}

\subsubsection{The SuperCDMS Experiment}

Now that we have a basic understanding of what dark matter is, we can look at how this candidate is being searched for. SuperCDMS is one of the leading direct detection experiments. This collaboration of scientists use a series of germanium detectors in order to search for WIMPs. The key advantage to SuperCDMS's experiment is its ability to detect low energy depositions from WIMPs, which many other experiments are unable to do.

The SuperCDMS experiment, like every experiment, has its own setup. The experiment is located deep underground (2341 feet) at the Soudan mine in Minnesota. Inside of the cavern there are a series of detectors set up into five separate columns known as towers. Each tower is comprised of a vertical stack of three detectors, used to look for a WIMP event. This configuration is helpful because it allows for cross referencing any potential WIMP events with other detectors, therefore providing a more accurate detection system. In addition, this experiment has much more finer details that add to its detection abilities that I will discuss momentarily.

One of the key ways SuperCDMS is able to detect low mass WIMPs is through a strong shielding process. Being deep underground allows for the majority of cosmic background particles to be blocked. It is important to block these cosmic particles, because they interfere with the ultra-sensitivity of the detector by causing background events in the detector. In addition the experiment has a three layer shield around the detector towers, made up of copper, lead and polyethylene. This additional shielding helps drastically reduce the rate of background electromagnetic events in the detectors. This is important because if these particles reached the detector one of two things could happen: the rate of background events would be so high that it would be impossible to distinguish a potential WIMP event or a false WIMP event would occur within the detector, which could the level to an embarrassing false WIMP detection.

The final piece that is important to mention about the SuperCDMS experiment is that it is operated at cryogenic temperatures to help allow for the materials used to become superconductors. In the current experiment the fridge 


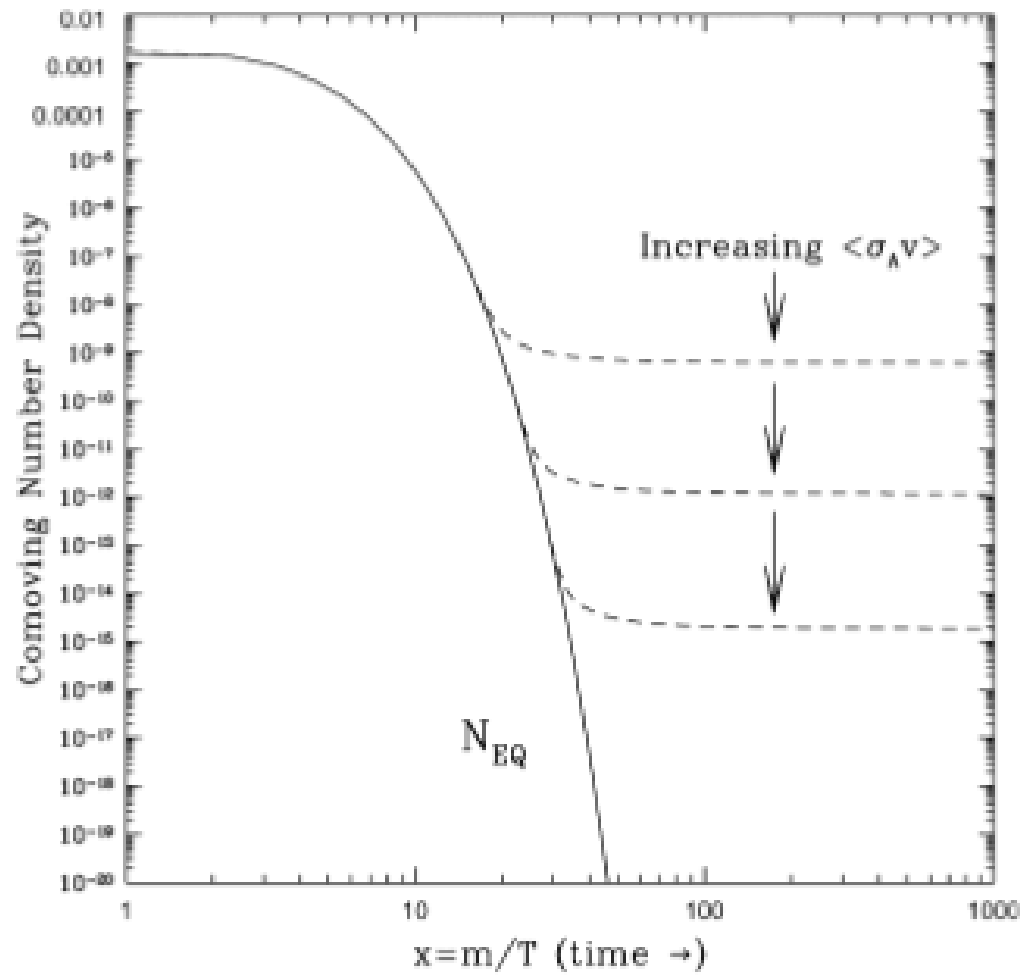

Figure 1.2: This graph shows what is known as the "WIMP Miracle", it details how cross-section and density interact. The WIMP miracle is an interesting phenomena that uses supersymmetric predictions for the initial amount of WIMPs and the natural decay over time to predict how much should currently be in our universe. This predicted amount of WIMPs almost perfectly matches the amount that we have been able to see experimentally from indirect detection. $[8]$ 


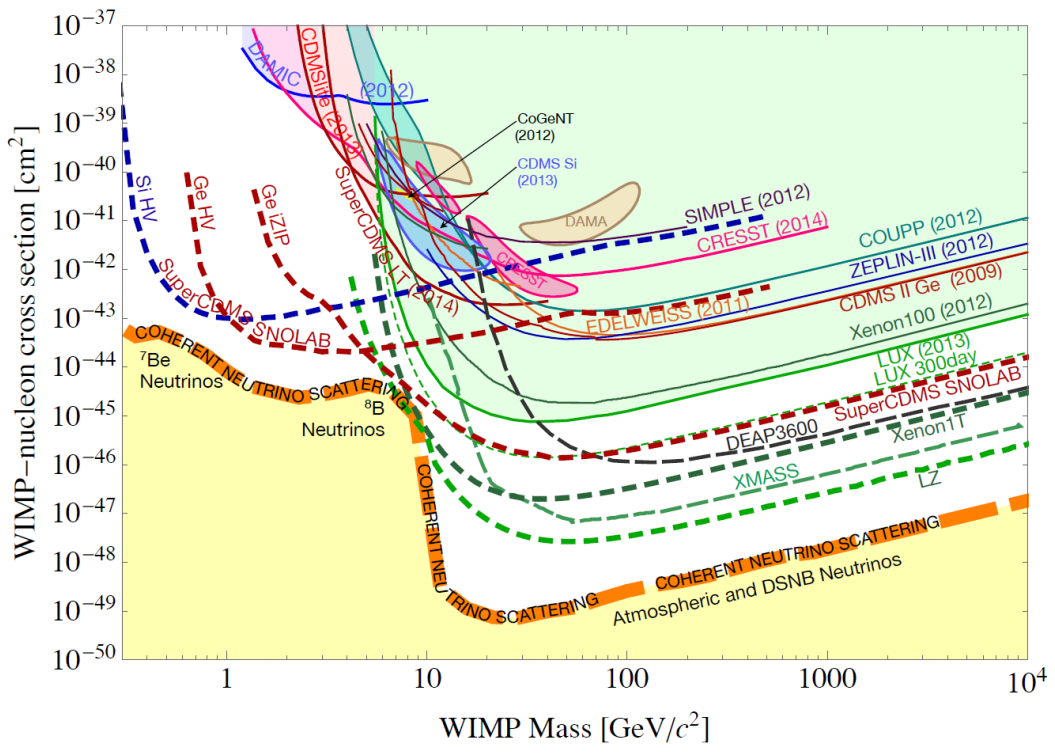

Figure 1.3: A model of many leading collaborations current and predicted detection ranges. The solid lines represent current detection curves, the dashed lines represent predicted detection ranges, and the bubbles show ranges where potential WIMP events have been detected. The dashed line at the bottom of the graph represents a natural barrier for the detection of WIMPs, as it's when neutrinos can cause interactions. As one can see SuperCDMS is the leader in low mass detection. [8] 


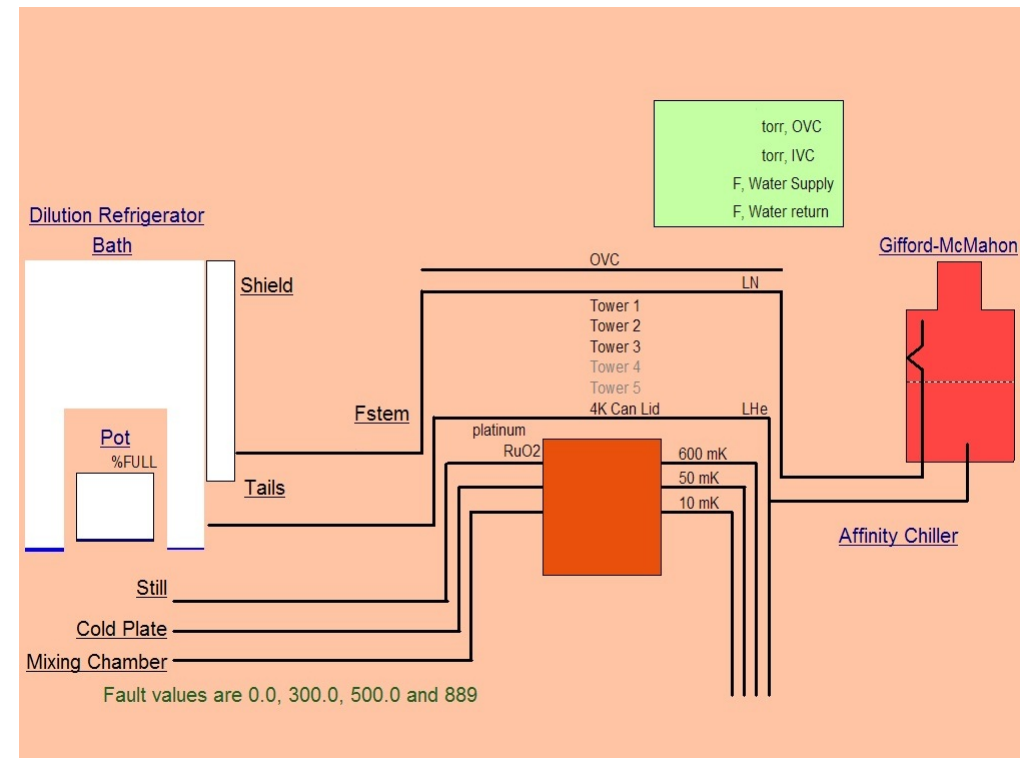

Figure 1.4: A highly simplified schematic view of only one component of the SuperCDMS fridge. As one can see the fridge is extremely complex

cools the cryostat to temperatures as low as 10 millikelvin. [8] In order to achieve temperatures this low the fridge require many components. Each of these components are extremely complex as can be seen in figure 1.4. Because of all these running parts, there is a chance that noise will be produced which will interfere with the super sensitive results of the experiment. In order to prevent this the collaboration has worked tirelessly to map out the major producers and mitigate them as much as possible. However, with the next generation experiment coming online soon, it is crucial to make sure there are no stray fridge variables producing any unexpected noise. This analysis, which is the subject of this paper, will be discussed in much more detail in section 3 .

\subsubsection{The SuperCDMS Detector}

The most unique and important component that SuperCDMS uses that allows it to target low threshold WIMPs is the sensor layout of its detectors. The detector is made of germanium and contains two sides that are each divided into four distinct sensors, designed to detect phonons that are produced by a WIMP-nucleon collision with a Germanium nuclei. [8]

The detector has many components which help with its search for WIMPs. One of these is the inner and outer charge electrodes located on both the top and bottom of the detector, which can be seen in figure 1.6. These electrodes are vital because they provide the voltage across the detector, but the purpose of 


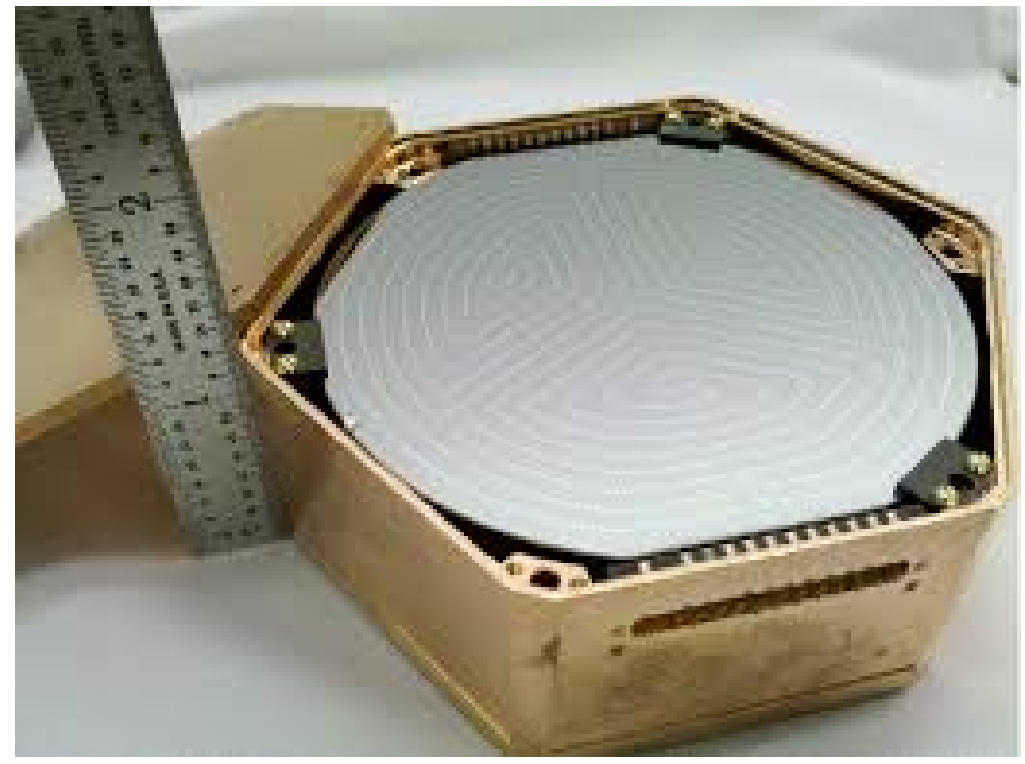

Figure 1.5: An image of an iZIP detector within its copper case. This detector has a $76 \mathrm{~mm}$ diameter and is $25 \mathrm{~mm}$ high. At the surface of the detector, the phonon sensors are clearly visible.

having two is more interesting. The reason there is an inner an outer electrode is to help discriminate against events that occur to far outside of the bulk of the detector. This is important because the copper shell of the detector is grounded so events that occur there will naturally have their charged particles travel to the walls rather than to the surface where they can be collected.

The next piece of the detector that is important to discuss is what is know as the phonon sensors. There are a total of four phonon sensors, three inner and one outer, on each side of the detector as seen in figure 1.7. These phonon sensors are integral for detecting potential WIMP events because they allow for being able to tell the difference between a nuclear recoil and an electron recoil. Being able to discriminate against electron recoils is vital because any potential WIMP event will be a nuclear recoil.

In addition the surface is lined with small sensors, called iZIPs, that allow for the collection of charged particles. These sensors are arranged in a specific pattern, which can be seen in figure 1.5. These iZIPs are what help make the detector so smart. The charge running through them allows the detector to collect electrons at one end and holes at another end as seen in 2.1. In addition this iZIP feature allows events that occur near the surface of the detector to be collected in a smart way. When a near surface event occurs the electrons and holes will split and be collected on the same surface side as seen in figure 1.8. This smart collection system allows SuperCDMS to help reject non-WIMP 


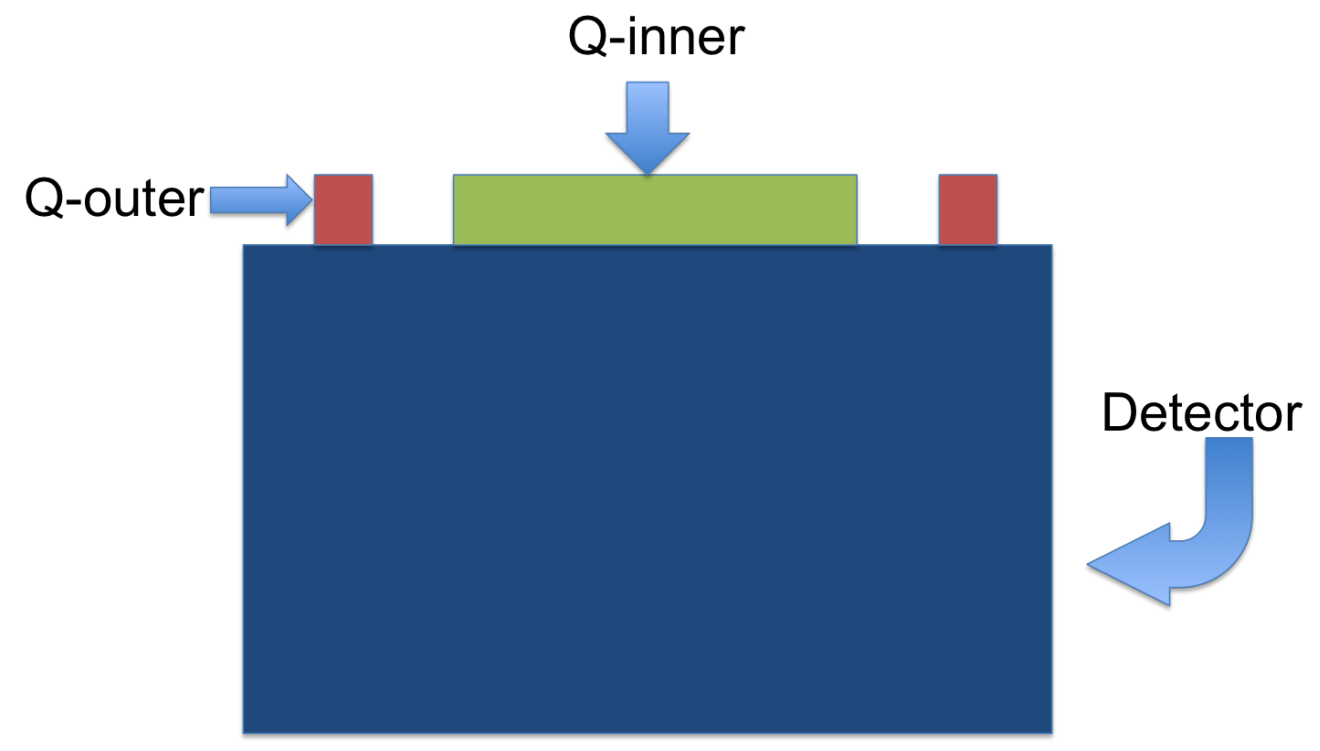

Figure 1.6: An schematic overview of a SuperCDMS detector. As can be seen the detector contains a Q-outer and Q-inner electrode.

events with great accuracy.

\subsubsection{The Importance of Background}

As mentioned earlier the SuperCDMS experiment is a highly sensitive experiment and requires extremely tight parameters in order to take data properly. One of the chief issues that the experiment comes up against is what is known as background noise (referred to simply as background). The most simple way to explain what background is would be that it is anything that is not wanted that interferes with the desired result. Imagine you are listening to music on your radio and all of a sudden you hear a bunch of static noise, this would be an example of background. The goal of our project in this example would be to reduce all of that static so that we can hear the music perfectly clear. Now that was a simple example but it helps illustrate the challenges of this objective.

So one of the most essential questions that needs answering is: what exactly causes this background in our experiment? Well the simple answer would be everything causes noise. Background is created by machines running, wires being plugged in and even simple vibrations from the ground. So since background producers are essentially everywhere, how is it that we could expect to get acceptable results? Well since we know background will always be present, essentially what it boils down to is reducing the noise to an acceptable level. This is done by placing machines in other rooms, using high-quality cables, 


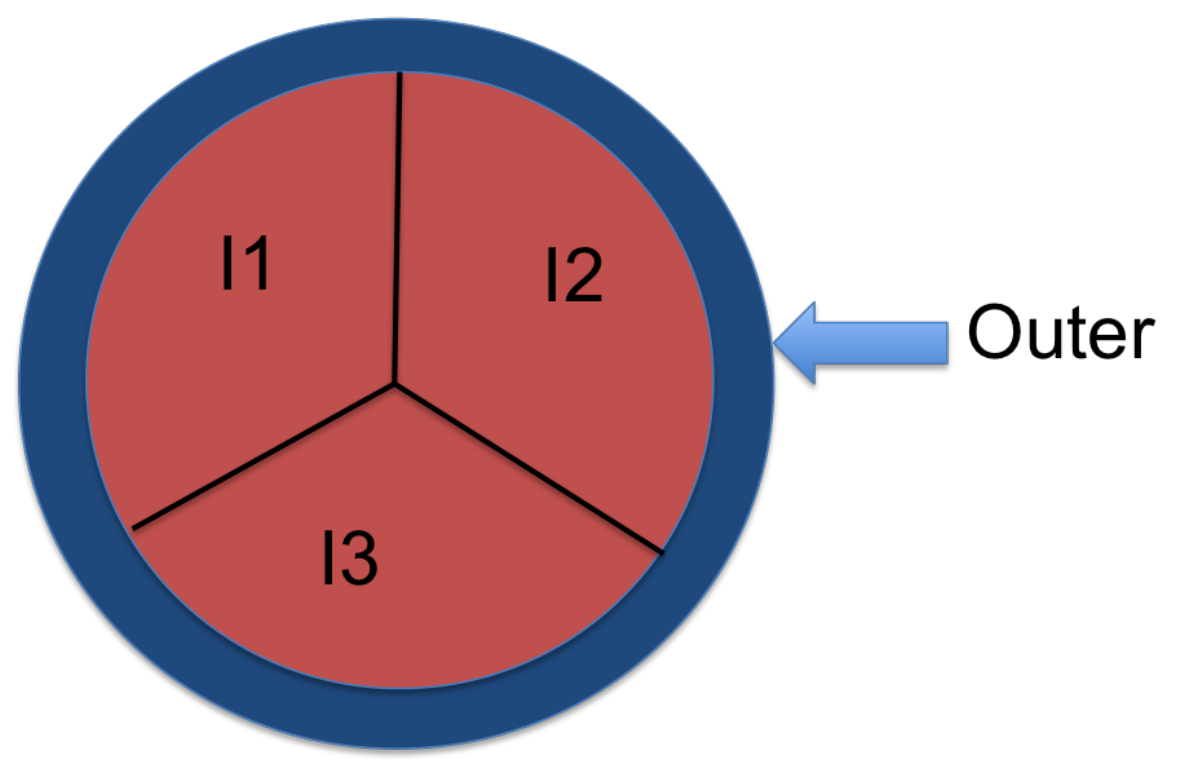

Figure 1.7: An schematic overview of the phonon sensors on one side of a SuperCDMS detector. As can be seen the detector contains 3 inner phonon channels and one outer. The reason behind the larger amount of inner channels is that the primarily targeted events are ones that occur within the bulk of the inner electrode. 


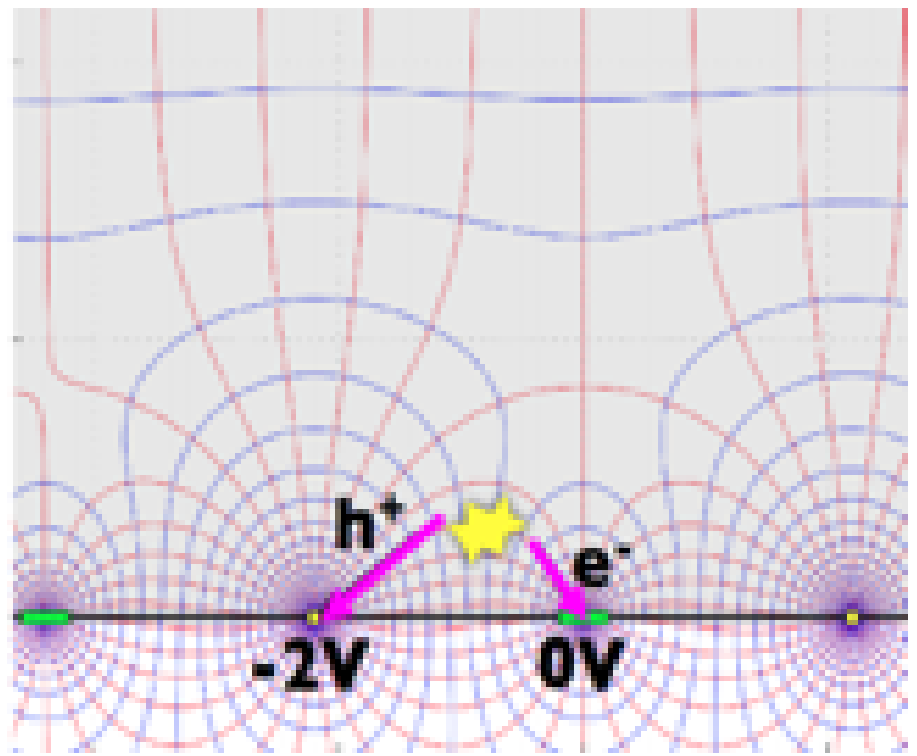

Figure 1.8: A modeled image of how an event occurring near the surface of an iZIP detector would react. As one can see that when an event occurs near the surface both the electrons and holes travel to the same surface side due to the electric field. This configuration helps discriminate surface events as they can lead to false nuclear recoil events. 
dampening vibrations, etc. Now while this sounds easy in theory it is actually quite a labor intensive process when put into practice. The reason behind this is because it is not always clear what the background source is. In order to identify the culprit most of the time it comes down to either trial and error or putting into practice an in-depth analysis. The former is good during the initial setup of an experiment but the latter can be much more useful for fine-tuning background sources when there is a large set of data available. This thesis will focus on an in-depth analysis of a large set of data that we have readily available.

Now it is also important to understand how background will manifests itself our results. Unlike in our example from earlier, it is not quite as easy as simply hearing a static noise. Background in detectors create readouts that have a very chaotic look, much like in figure 1.9. However, when there is very little background it is much more clear what the result will look like. Because of this it is important to reduce background levels to as low of level as possible. It is important to note though that in the SuperCDMS experiment there are low levels of noise with enables detection of low energy events, resulting in small peaks.

Sometimes reducing background levels to as low of a level as possible is not enough to provide as accurate of results that are required. In order to solve this issue computer scientists will create programs that filter the data and cut out as much of the background that can safely be removed. This process is known as cutting the data. Now while cutting is useful, there are some drawbacks. If the cut is not performed properly it can make the actual data look much differently than it actually does. This can lead to false claims of success or even missing real data events. Therefore it is vital that the cuts that are placed on the data are accurate.

The final piece that should be elaborated on more in this brief introduction to background is how it relates to the project that is being discussed in this thesis. The second experiment that is discussed in chapter 3, is an attempt to correlate the SuperCDMS fridge variables with detector noise levels. Essentially what this boiled down to is scanning data from the fridge and comparing it to results that were taken at the same time from the detectors. This is important to mention now because it is an example of suppressing background levels in experiments. Suppressing noise requires large sets of data to be able to work, but it allows you to potentially view background sources that were not discovered in the initial analysis. In addition this process is important when trying to preemptively scale a current experiment into a future one. This is useful because it allows you to impose even tighter restrictions on your noise that are not currently in place on the current experiment.

As one can see understanding and decreasing background noise is a crucial element to any experiment. If it is not properly planned for it cause poor sensitivity. Therefore it is crucial to take proper steps to identify and fix any potential background culprits. 


\section{Zip 5 Phonon}

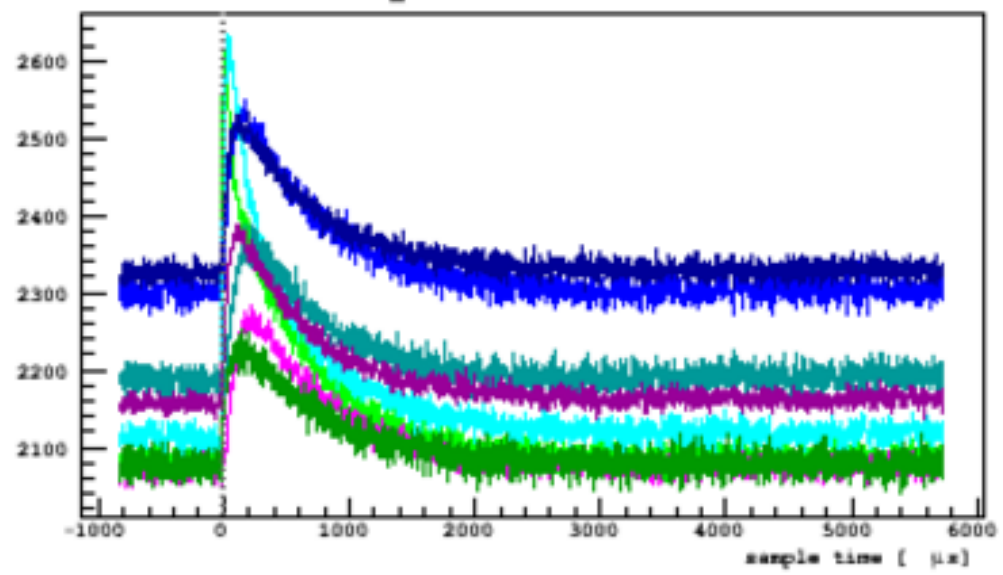

Zip 5 Charge

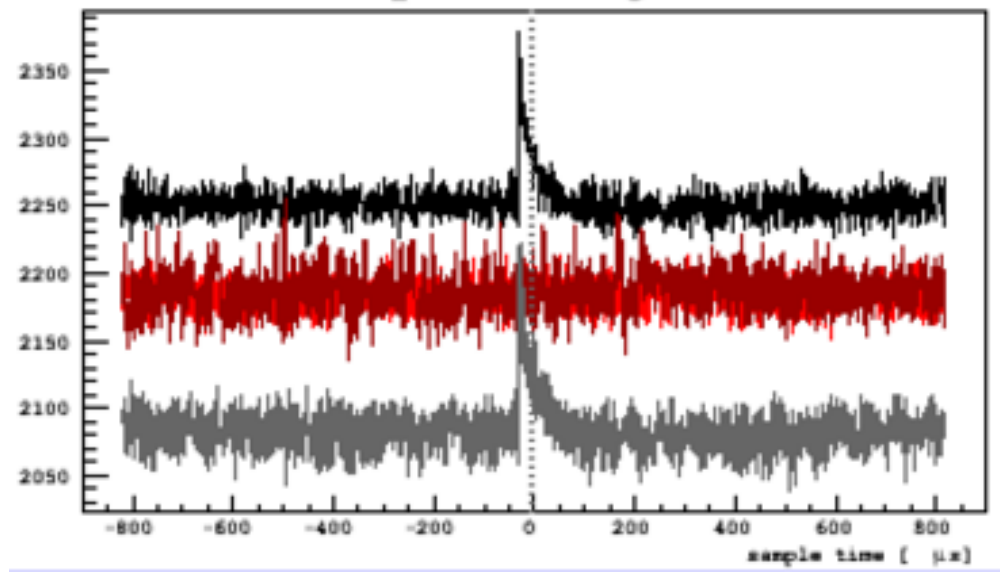

Figure 1.9: An image of channel from the SuperCDMS experiment. As can be seen in this image there is a large amount of background within the center channel on the bottom graph, which can be determined by the large fluctuation. Note that the large peak seen on both of these graphs is an event. 


\section{Chapter 2}

\section{Simulating Propagation of Charged Particles in the SuperCDMS Detector}

In this chapter I will discuss my efforts to take simulate charged particles propagation through germanium detectors. Understanding how these particles propagate, enables us to better understand what the limits of our detectors are and how detector charge performance could be improved. Therefore, in this chapter we will examine how this simulation was performed and the insight it has provided into the plausibility of potential future detector designs.

\subsection{What and Why}

One of the hardest things to do in experimental particle physics is to visualize what you are studying. In order to tackle this problem many physicists will create what is known as simulation packages to study what is going on in their experiment. In addition, simulation allows scientists to test experiments that would be otherwise virtually impossible. By creating a simulation that contains all the known physics, scientists can simulate experiments in order to better understand and predict detector performance with great accuracy and at relatively low cost before their implementation. In this section we will discuss how simulation is intended to help determine if new and better types of detectors are possible.

One of the most important pieces to any experiment is making sure you know how your machinery or detectors work. However, it can be very difficult

to fully understand how a detector works on the atomic level. In order to solve this issue, collaborations like SuperCDMS have created state of the art simulation packages that model the underlying particle interactions within a medium. These simulation packages can be complex and account for almost every known 
relative aspect of the detector and physics process. Realistic simulation is used to create the most accurate results as possible. One of the simulations that was used by SuperCDMS was created within the Matlab environment. In it, the user can do a variety of tests, but I use it to focus primarily on what happens during an event in a germanium detector.

The primary goal of this test was to help probe whether or not similar detectors could be operated at higher temperatures than the current SuperCDMS operation temperature of about $40 \mathrm{mK}$ and still maintain the ability to differentiate between electron and nuclear recoils within the detector. If the temperatures could be raised to $77 \mathrm{~K}$, without compromising the differentiation, then the whole experiment could be operated at a greatly reduced cost. The reason behind this is because trying to achieve the cryogenic temperatures that SuperCDMS requires takes a great deal of machinery, all of which cost large amounts of money. If a detector could operate at warmer temperatures, the amount of materials required in order to achieve that temperature would be greatly reduced, thus decreasing setup and operational costs. However there are many challenges trying to achieve this goal. The main challenge is that when the SuperCDMS detector is warmed up even a few degrees Kelvin, its performance deteriorates quickly. Due to this a new detector entirely would need to be designed to achieve this goal. Therefore, it was crucial to perform this type of simulation to see if this goal is even worth feasible.

\subsection{The Simulation}

So now that we know the goal of my simulation effort, let's look into detail on how it was actually performed. I was provided a working simulation package developed by a Stanford graduate student that utilized the Matlab environment. Naturally the first step was to explore the simulation package and become familiar with how it worked. Once the simulation was installed, I set about figuring out how to properly run the simulation. First I ran the standard simulation to make sure the results matched that of the manuals. The image I first obtained is pictured in figure 2.1. First, I noticed the obvious things were correct like that there was only one event and that the event occurred at the proper location. I also noticed the holes propagate upwards and the electrons propagate downward, which is due to the upward facing electric field, so this was also correct. In addition to the proper propagation direction, I noticed that the electrons spread out much further than the holes, which is again expected (the actual reason for this will be discussed later). Therefore due to all these factors, I was able to confirm that the simulation I ran was showing correct results.

Once this goal was achieved, I next began trying to manipulate different variables within the simulation. This step was difficult at first because it took time locating where each variable was located within the program. I discovered that almost every variable could be manipulated but for this step I simply tried to move where the event location happened within the bulk of the detector. I tested the event location with multiple locations. One example of my results 


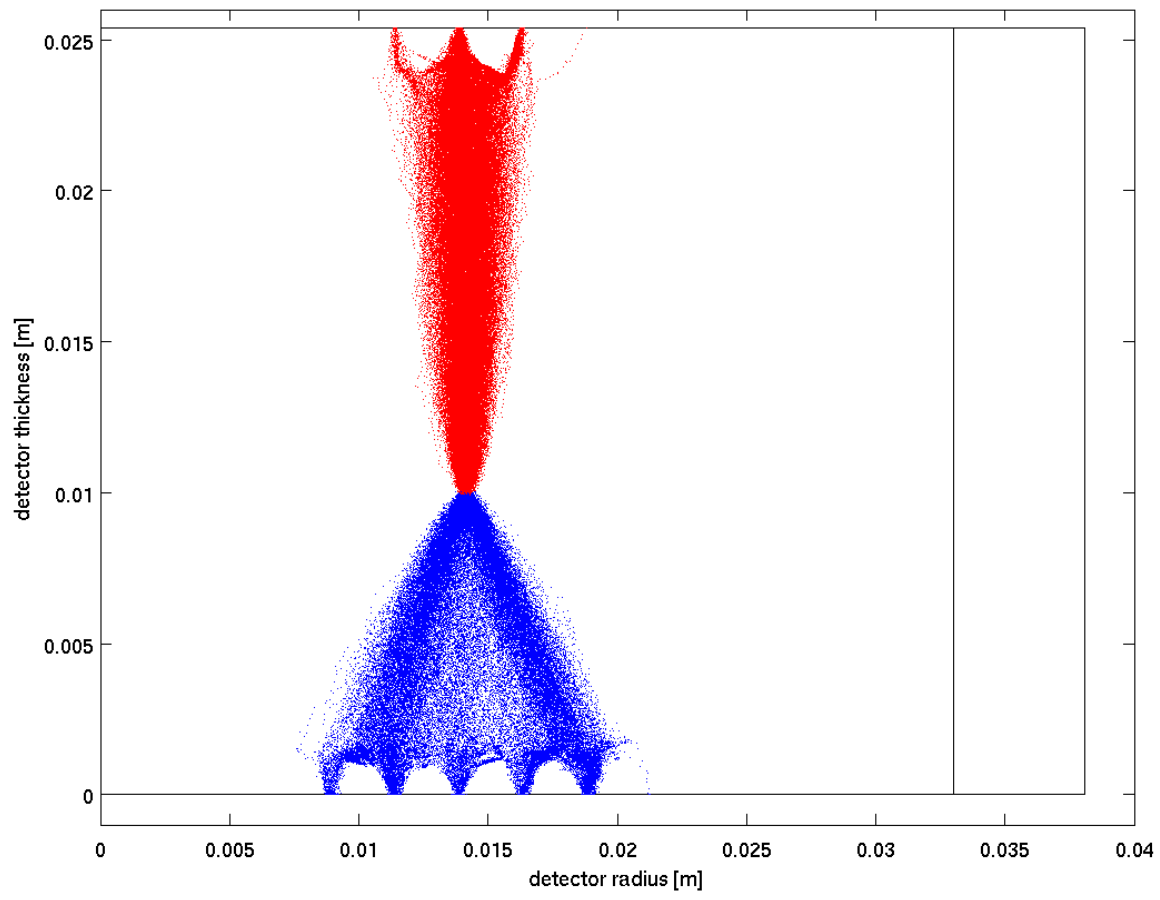

Figure 2.1: A simulated image of an event occurring in an iZIP detector. The event occurred at a radial position of $.015 \mathrm{~m}$ and at a height of about $1 \mathrm{~cm}$ above the bottom of the detector. The vertical line at a radial position of $0.032 \mathrm{~m}$ denotes the boundary between the portion of the detector covered by the inner charge electrode and that of the outer electrode. Holes propagate upward following the electric field and electrons have a smaller effective mass and spread out more as the propagate down towards the bottom of the detector. The surface electric field described in section 1.3.2 causes the arching feature as they approach the top and bottom of detectors. Here the holes are represented by the lighter color traveling towards the top of the detector and the electrons are shown in the dark color traveling towards the bottom. 
using different event locations can be seen in figure 2.2. As can be seen the major factors: like propagation direction and number of events are all the same as the first event shown in figure 2.1. The only thing that changed was the initial event location and spread size, which is a direct result of the different event location. Once I saw that all my results matched those of the manuals, I knew my simulation was working properly.

It is also important to note that I set some important parameters during this step. The initial package modeled a standard SuperCDMS iZIP detector (described in detail in Section 1.3.2) with the option of choosing between germanium or silicon as the material that makes-up the detector (for the purposes of this experiment, only the germanium detector was used). In addition, important values for variables like the bias voltage on the surface of the detector were left at their original set values. All of these can be found in the simulation manual [? ].

\subsubsection{Testing the Simulation}

The next step in this process was to begin to start running tests with this simulation so that we could determine whether or not the spread (this term refers to the radial spread size of the charged particles at the surface of the detector) could be manipulated in a way that would prove a new type of detector could be viable. The spread is a great indicator because it differs greatly between different types of events i.e. nuclear and electron recoils. If a significant change in one of the current parameters in the SuperCDMS detectors could be determined to make a significantly large enough difference, then it could potentially mean a new type of detector could be conceivable.

The first test that was run was simply how does the position of the event within the detector effect the spread. As mentioned in the previous section I left important variables like bias voltage $(+2 \mathrm{~V})$ at their standard fixed values in order to make sure I was only testing the event position. I then went about testing how spread was effected by initial event location within the detector. When looking at the results of this test, as can be seen in figure 2.1, I noticed that electrons seem to spread out much more quickly than the holes do. This phenomenon is to be expected because of the low electric field and temperatures used in this experiment. At these conditions electrons are located in the L-band ground state [111], which makes their travel much more complex than the holes which are in the Gamma ground state. [3] Due to this electrons suffer from an anisotropic energy-momentum. This anisotropic energy-momentum is given by:

$$
\epsilon_{\text {electron }}(k)=\frac{h^{2}}{2\left(k^{2} / m_{1}^{2}+k^{2} / m_{2}^{2}\right)}
$$

where $\mathrm{k}$ is the wave number, $\mathrm{h}$ is the Dirac constant, $m_{1}$ is the longitudinal mass and $m_{2}$ is the transverse mass. The ratio between the longitudinal and transverse mass is approximately 19.5. [3] A similar calculation can be done for holes: 


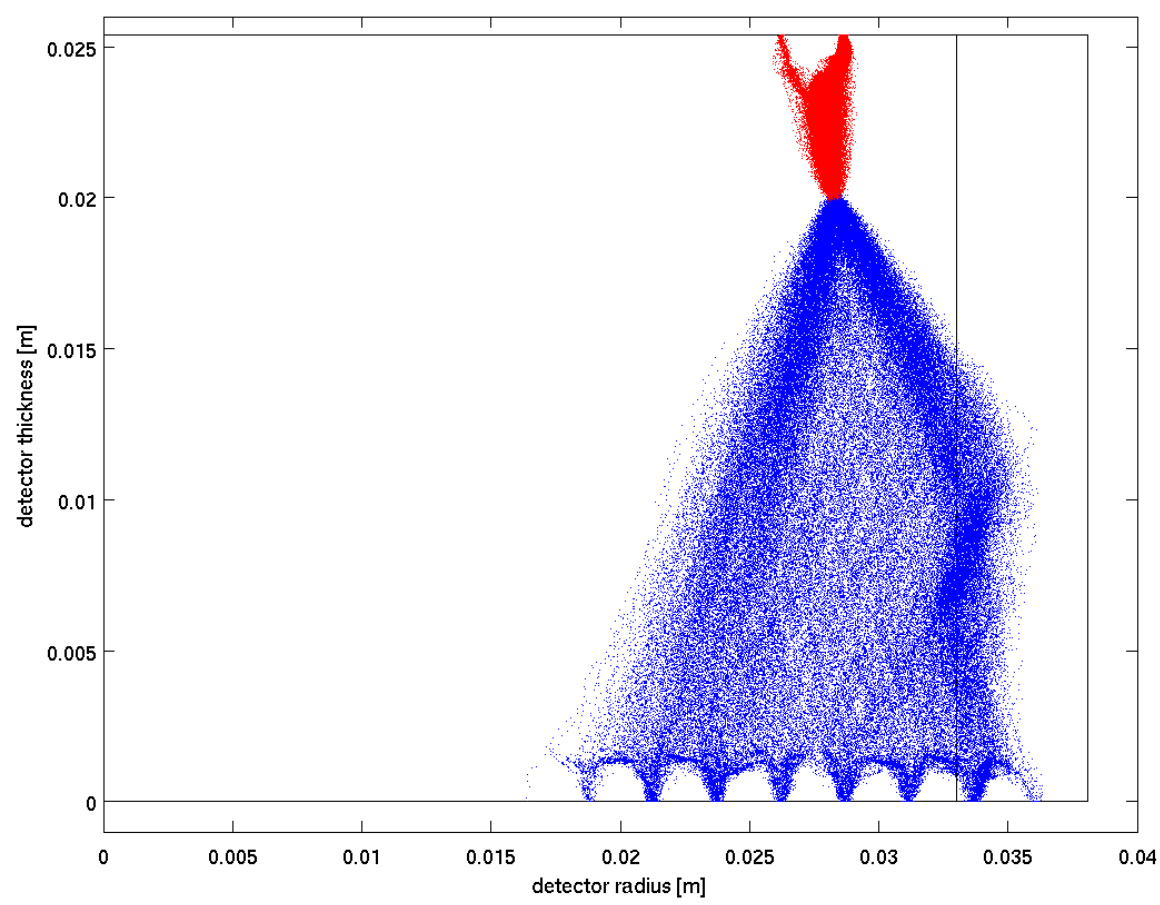

Figure 2.2: A simulated image of an event occurring in the bulk of an iZip detector. The simulated image is similar to that in shown in figure 2.1, except its initial event position was changed to $(.03, .02)$. Here the holes are represented by the lighter color traveling towards the top of the detector and the electrons are shown in the dark color traveling towards the bottom. It is interesting to note that as the electrons hit the boundary between the inner and outer charge electrode their spread is effected. 


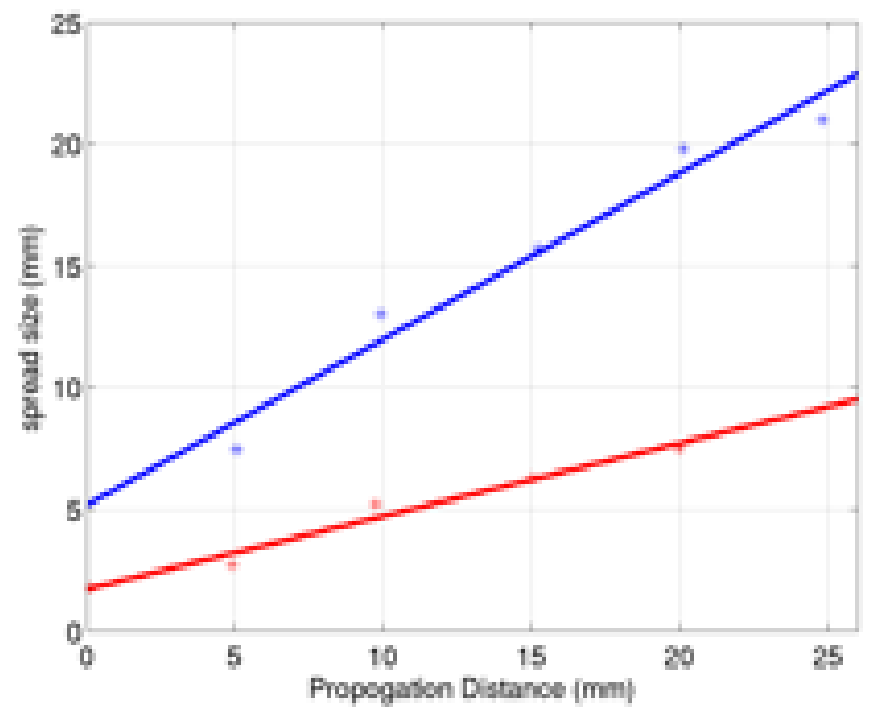

Figure 2.3: A graph detailing the simulated spread difference based on the event location in the detector. Electrons are shown by the dark color and have greater spread. The holes are shown as the lighter color and contain a lesser spread.

$$
\epsilon_{\text {hole }}(k)=\frac{h^{2} k^{2}}{2 m}
$$

where $\mathrm{k}$ is the wavenumber, $\mathrm{m}$ is the mass and $\mathrm{h}$ is the Dirac constant. It is clear by this equation and the fact that holes are in the $\gamma$ state that there is very little $\mathrm{x}, \mathrm{y}$ movement when related to $\mathrm{z}$ movement. This shows that theoretically the electrons spread much more quickly than the holes do, so to see this result in the simulation proved that the simulation was behaving as one would expect. It is also important to note that SuperCDMS experimentally observes this result as well.

The next step was to scale up my simulation and test numerous different initial event positions. In order to do this, I ran several additional simulations in which a different radial position and depth within the detector was chosen. In order to be thorough and consistent, I first tested only the radial location and fixed the depth at $.015 \mathrm{~m}$. The results of this test showed that the spread size was nearly identical when the event occurred within the bulk of the detector. The next logical step was to fix the radial location (location was $.015 \mathrm{~m}$ ) and vary the depth. I found that the spread of these results varied so I recorded the results, which can be seen in figure 2.3. By looking at this plot I can see that the trend for how spread changes generally followed what was to be expected, which was another sign that the simulation was behaving as one would expect it 


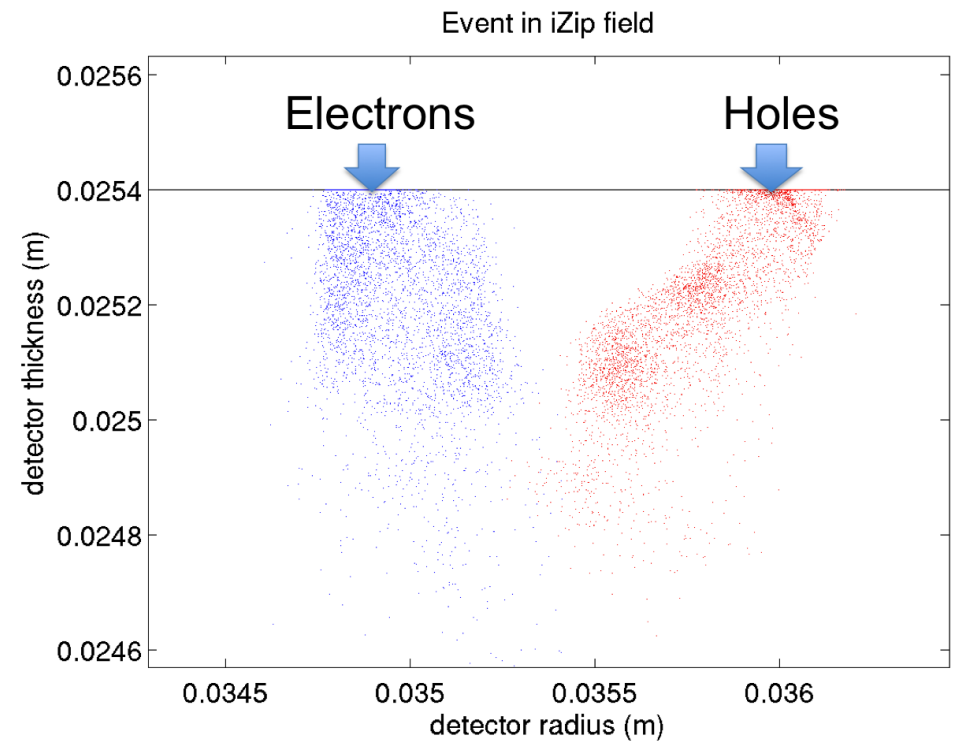

Figure 2.4: A simulated image of a surface iZIP event. This image is zoomed into the center of the detector. The electrons are shown moving to the left and the holes are shown moving to the right. Note: the surface of the detector is located at $.0254 \mathrm{~m}$, the extra space above this point is outside the detector.

to. This meant that the simulation was working well and the underlying physics appeared to be correct. Therefore I was ready to proceed on to the next test I would conduct.

\subsubsection{Unusual Propagation Features}

While testing the charge propagation, I found two cases where an event would propagate in a surprising way. The first was when an event occurred at the top or bottom surface of the detector. Now as I mentioned earlier in section 1.3.2, iZIP detectors contain a special feature that help deal with events occurring on the top or bottom surface of the detector. When an event occurs on one of the surfaces, the electrons and holes propagate following the electric field to only one side of the detector, which can be seen in figure 1.8. Due to this feature the holes and electrons will not propagate in the same way as an event occurring in the bulk of the detector. This is intentional to enable discrimination between surface events and those occurring in the bulk of the detector. The reason behind this is because nuclear recoils can be faked by surface events, which could cause a fake WIMP events. Therefore we can say that the simulation of the charge propagation of surface events does model actual events and therefore it does behave correctly. 


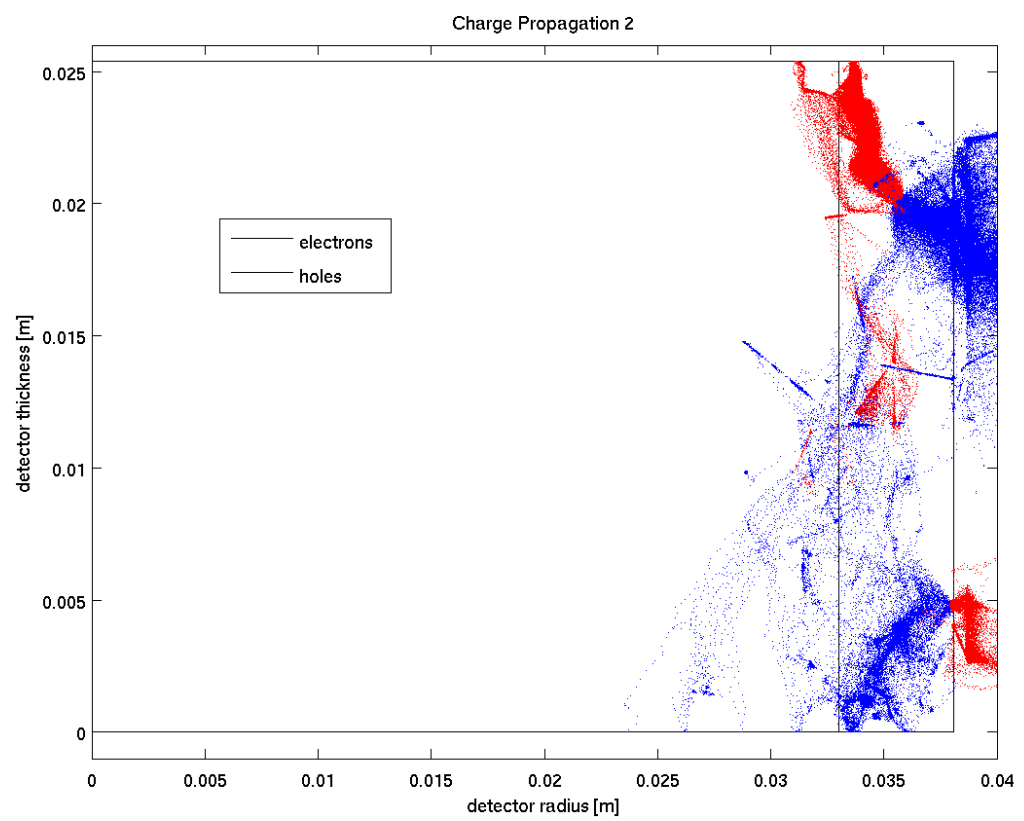

Figure 2.5: An image of two simulated events occurring within the outer electrode. As one can see the charged particles propagate towards the side of the detector which is where the grounded copper shield is located. The dark color represents the electrons and the holes are represented by the lighter color.

The second type of event that seems to propagate in an unusual manner is one that occurs near the boundary between the inner and outer charge electrode of the detector, which was described in section 1.3.2. When an event occurs here, the electrons or holes, seem to be effected by the boundary, which can be seen in figure 2.5. The reason behind this may seem simple, but there is actually another factor that is causing the shift. The copper shield of the detector is grounded so the charged particles will naturally travel towards the side when close enough. The maximum distance from the sides that this seems to occur is about where the inner and outer electrodes split. It is also important to note that this is observed by SuperCDMS in their data. Therefore, because we know this is a natural and known property of the detector, we can say the detector is behaving as it is expected.

As one can see these two abnormal cases that were found in the simulation are both expected to occur under these conditions. Therefore we can say that this simulation behaves as we would expect for all tested situations. 


\subsubsection{The Effect of the Electric Field on Charge Propaga- tion}

Another key component that this simulation allows us to control is the charge bias across the detector. This is important to test because as mentioned in section 2.2 , the detector could be completely redesigned. If we could prove that the electrons and the holes spread at a similar rate with a very low voltage as they do with a high one, then it would be reasonable to say that a new type of detector technology could be developed to help increase accuracy. This would be a huge advantage because it would greatly reduce the amount of money that is needed to operate the experiment. In addition, if this was feasible it would allow us to help better differentiate between nuclear recoils and electron recoils by comparing the size of the charged particle's spread. Now, in order to change the bias, I changed the bias in the simulation's code to different reasonable levels.

In my project I first tested two extremes, one high voltage and one low voltage, as well as an intermediate voltage. In addition the initial event location was fixed at the $(.01, .01, .01) \mathrm{m}$ position (the origin is located at the very center of the bottom face of the detector). This means the event was rather close to the surface detector meaning it would naturally have less time to spread out. This fixed event location was randomly chosen and it would be completely acceptable to pick any other location within the bulk of the detector. In order to analyze how the voltage was affecting the charge propagation I looked primarily at how far the electrons and holes spread. For the intermediate voltage trial run, the electric field strength was set at $1 \mathrm{~V} / \mathrm{cm}$ of detector thickness. This bias level is very close to the $2-3$ volts of total voltage that are normally applied across an actual SuperCDMS detector. I found that the electrons spread on average $3.54 \mathrm{~mm}$ per $\mathrm{cm}$ of distance traveled while the holes spread $3.84 \mathrm{~mm}$ per $\mathrm{cm}$ of distance traveled. The next trial that was ran was the extreme low voltage. For this trial the voltage was set at $.02 \mathrm{~V} / \mathrm{cm}$ of detector thickness. After collecting the results for this trial, I determined that the electrons had an average spread of $4.44 \mathrm{~mm}$ per $\mathrm{cm}$ of distance traveled, whereas the holes spread $5.08 \mathrm{~mm}$ per $\mathrm{cm}$ of distance traveled. An example of what one of these simulations looked like can be seen in figure 2.7. When the high voltage trial $(50 \mathrm{~V} / \mathrm{cm})$ was simulated, it was observed that the electrons spread an average of $1.27 \mathrm{~mm}$ per $\mathrm{cm}$ of distance traveled and the holes spread $2.62 \mathrm{~mm}$ per $\mathrm{cm}$ of distance traveled. An example of one of these simulations can be seen in figure 2.8 .

The next step was to expand this test to include more intermediate voltages as well as even more extreme voltages. The results of this trial can be seen in figure 2.6. As one can see the spread size of both electrons and holes are inversely proportional to the strength of the electric field. It is also interesting to note that the spread seems to be linear for the electrons, whereas the holes seem to lose their linearity when the voltage is very low. This may be caused by the initial event location that was chosen, which was very close to the bottom surface. Due to this the holes had a much larger distance to travel, so in an extremely low voltage situation they could be affected by the low pull of the 


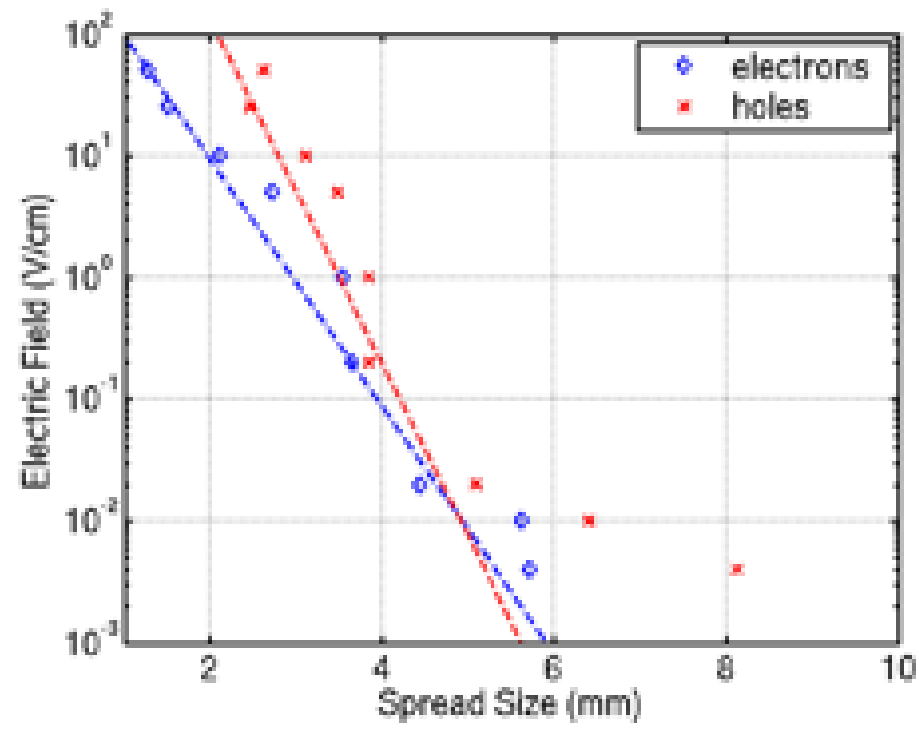

Figure 2.6: A plot that shows how voltage effects propagation. As usual the dark color represents electrons and the lighter color shows holes. As one can see the holes have a linear tendency when voltage is being varied. However holes do not contain this tendency and seem to exponentially increase in spread size as the field decreases.

electric field, making them more likely to travel along the crystal lattice.

The reason that the spread was much smaller in the high voltage compared to the others is that electric field provides a greater upward/downward force on the electrons and holes causing them to travel in the $\mathrm{z}$ direction quicker. In addition holes are less influenced by oblique propagation following the Ge crystal lattice, causing them to spread less. Since the ions are being pulled harder and are thus traveling quicker, they have less time to spread out. Therefore, by varying the electric field in the SuperCDMS simulation, I was able to confirm that the higher voltages cause electrons to spread less.

Using these results we could help optimize the voltage to cost ratio the experiment uses. In addition, this could help differentiate between a nuclear recoil and electron recoil. The way this could be done by taking the relative spread size of each charge particle for both types of recoils and using that data to in combination with the sensors on the surface, more accurately differentiate between the two. This could be useful because it would increase accuracy and could help more quickly reject non-events.

In addition we can use these results to determine if the spread at high voltage would be small enough to potentially enable discrimination between electron and nuclear recoils. We can use the typical absorption length of photons shown in 


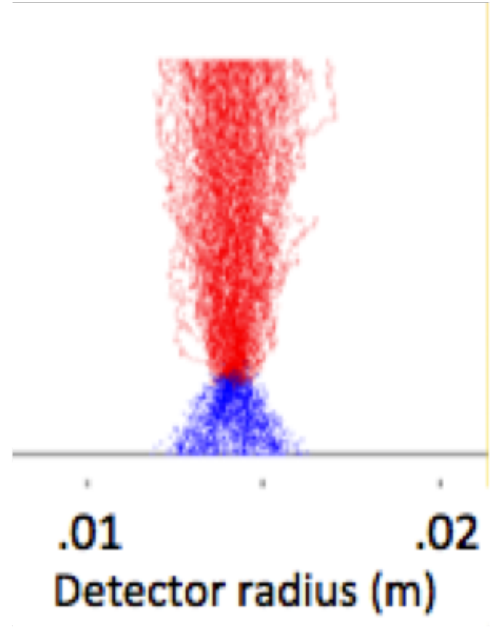

Figure 2.7: A zoomed in image of a simulated event occurring in at the initial location $(.01, .01, .01) \mathrm{m}$ in a standard CDMS detector. Here the holes are represented by the lighter color traveling towards the top of the detector and the electrons are shown in the dark color traveling towards the bottom. This low voltage (.02 V/ cm of detector thickness) test shows the spread is very large and relatively messy.

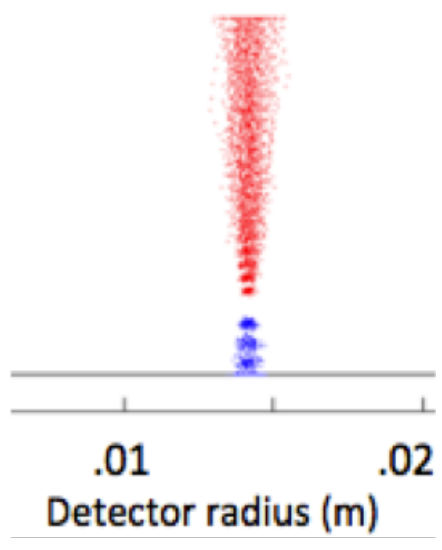

Figure 2.8: A zoomed in image of a simulated event occurring in at the initial location $(.01, .01, .01) \mathrm{m}$ in a standard CDMS detector. Here the holes are represented by the lighter color traveling towards the top of the detector and the electrons are shown in the dark color traveling towards the bottom. This high voltage $(50 \mathrm{~V} / \mathrm{cm}$ of detector thickness) test shows the spread is very small and clean. 


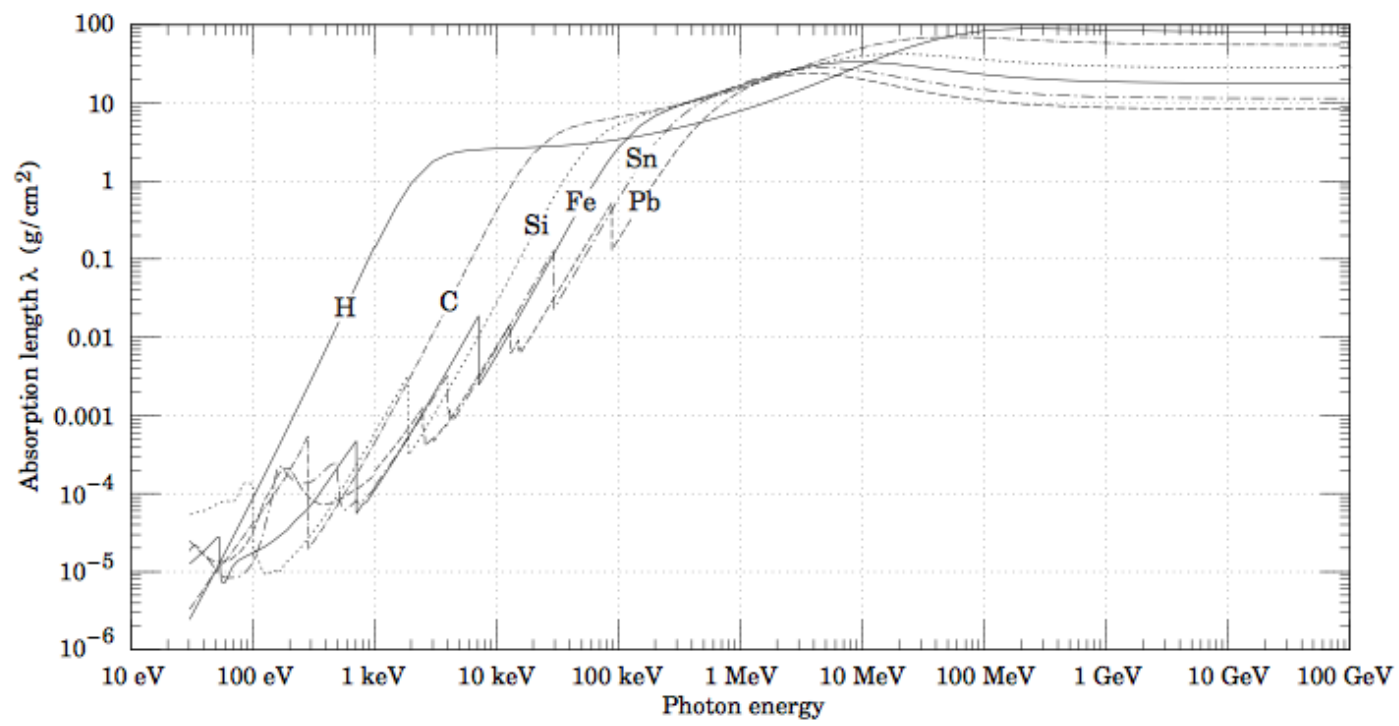

Figure 2.9: A graph from the PDG that shows the absorption length at different photon energy levels. As one can see elements like Si that contain a similar density as Ge are shown in this graph. This graph can be used to see whether or not a normal event within a SuperCDMS detector could travel far enough to reach the sensors at the surfaces.

figure 2.9 to estimate the initial spread of the deposited charges for an electron recoil.In contrast, the initial spread of the deposited charges for a nuclear recoil is about $20 \mathrm{~nm}$. Now when comparing this with the size of the SuperCDMS detector we can tell that the charged particles from a bulk event would be well absorbed before they reached the surface. Therefore, this test helps us prove that it would not be worthwhile pursuing turning up the voltage for a detector the size of the current SuperCDMS detectors.

As one can see the use of voltage is a crucial part of how particles propagate throughout a germanium detector. Understanding how it effects the propagation has huge ramifications on the efficiency of the experiment as a whole. Therefore this test was crucial to help understand the ramifications of how the potential of changing the voltage could be useful.

\subsection{Chapter Summary}

As one can the use of simulations can be useful to help gain information about experiments. In specific, simulations were crucial in order to test the feasibility of ideas that could be implemented in the future. As one can see this chapter showed that holes and electrons will spread at different rates in Germanium 
detectors. This is important note because the size of their spreads makes unrealistic to try and place the charge sensors close enough to try to use the measured spread to distinguish between nuclear and electron recoils. In addition, this chapter proved that spread size is directly correlated to the size of the charge bias. 


\section{Chapter 3}

\section{A Systematic Quantification of SuperCDMS Noise}

The second project that will be discussed in this thesis is my attempts to quantify the level of noise in the detectors caused by the SuperCDMS fridge. As will be discussed later in this chapter, the motivation behind this experiment is to help reduce any potential background noise that is produced by the fridge for the next generation experiment at SNOLAB. Therefore, in this chapter we will examine how a data analysis experiment was designed and implemented to help find any unknown noise culprits.

\subsection{What and Why}

It is important to be able to identify the level of background noise within the experiment and be able to reduce it to a level that is considered acceptable. In order to do this, it takes detailed studies to identify these potential noise culprits and then someone going in and trying to fix the noise sources. In this experiment I attempted to find a way to systematically quantify the levels of background within the SuperCDMS detectors to try and find any background noise culprits that were not known to the collaboration.

One of the main reasons this is an important type of test to conduct is because background noise can have serious effects on the potential results. As we discussed in section 1.3.3, background noise can cause issues like missing potential WIMP events or even triggering false WIMP events. Due to this it is crucial for us to search for ways to reduce these background levels as much as possible. In addition, the next generation experiment at SNOLAB will be much more sensitive than the current experiment. Due to this, background noise levels will need to be lower than their current levels. Therefore it is vital to fix any obvious noise producers.

So where and how is all of this background noise produced? Well one of the main contributors in the SuperCDMS experiment is the dilution fridge and 


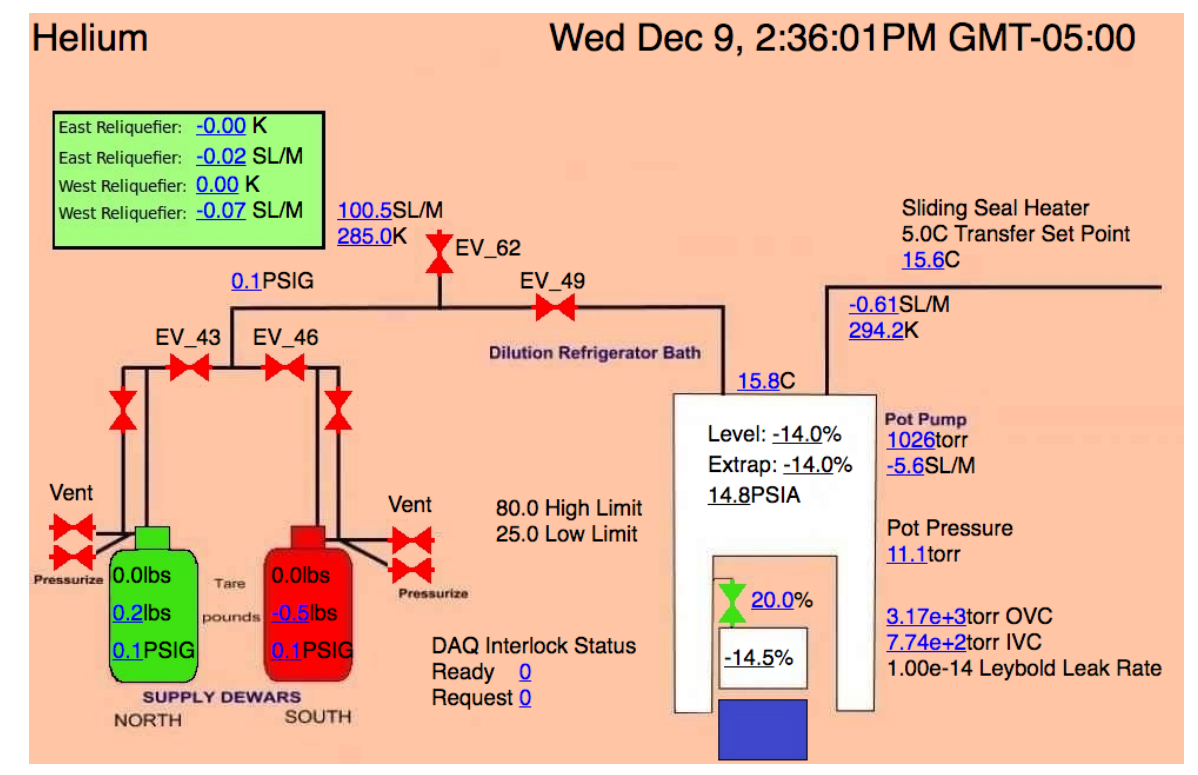

Figure 3.1: A live schematic snapshot of the SuperCDMS helium chamber. This image is just one of the many components that make up the SuperCDMS fridge. As one can see the fridge is very complex which greatly increases the chance of background noise being introduced to the experiment. Therefore it is crucial to try and minimize this background noise as much as possible. [8]

cryocooler. A dilution fridge, commonly referred to as a slow fridge, is one of the most efficient ways to cool to the cryogenic temperatures (around $40 \mathrm{mK}$ ) that are required for this experiment. This fridge contains multiple chambers, two of which can be seen in figures 3.1 and 3.2, that work to help produce this super cool temperature. However, due to all these moving parts and required pumps, the chances for background noise increase dramatically. Therefore it makes sense to design an experiment in which we search to see if any of these parts are causing above desired noise levels.

Finally it is also important to mention another important piece of the fridge's mechanics. The SuperCDMS fridge records the value of all of its variables every three seconds in order to have an accurate record of what was going on at the time data was being taken from the detectors. However due to these frequent snapshots, there is an incredibly large amount of data that must be sifted through making this type of experiment much more difficult. 


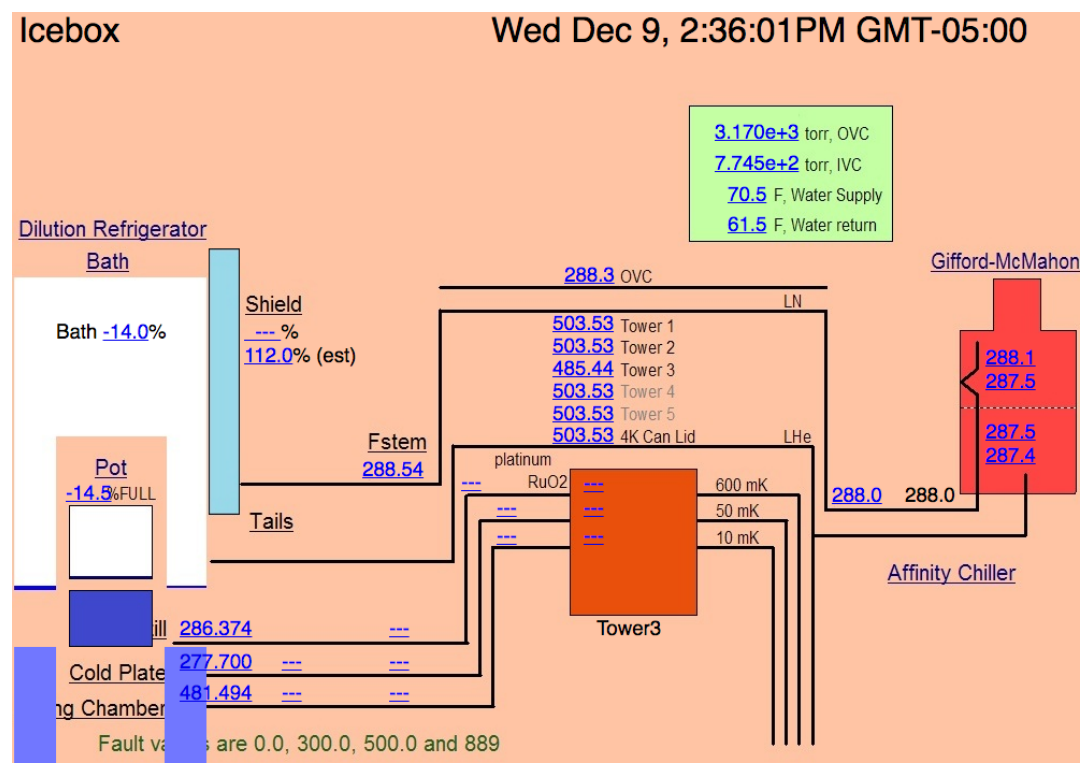

Figure 3.2: A live schematic snapshot of the SuperCDMS icebox. Like figure 3.1, this image is just one of the many components that make up the SuperCDMS fridge. As one can see this fridge is very complex which increases the chance of background noise being introduced to the experiment. Therefore it is crucial to try and minimize this background noise as much as possible. [8] 


\subsection{Fridge Parameter- Detector Noise Study}

\subsubsection{Setup}

To begin my analysis I was given two sets of data. The first of which was dilution refrigerator data, stored in a remote MySQL database, and the second was the detector data, stored in the SuperCDMS's Matlab based analysis framework. So my first goal was to learn how to access each set of data. The first one I attempted to work with was the SuperCDMS data, because it simply required knowledge of Matlab to access, which I was familiar with from the simulation described in chapter 2. In order to access this data I learned that I needed to install SuperCDMS's analysis framework CAP, which gave me access to the SuperCDMS data. I tested accessing data from this source multiple times and compared the data series numbers with the online SuperCDMS web page. Once I was able to confirm that the series numbers (this is the way SuperCDMS keeps track of each piece of data that was taken from the detectors) on the data I had accessed matched the web page's data I was able to move on to trying to access the fridge data from the MySQL database.

The next step was to try and pull the fridge data from the remote MySQL database. This step proved to have many challenges because I was unfamiliar with how MySQL worked. So I set about learning some of the essential commands that make MySQL work. In specific, I learned commands that allowed me to access data. Once I felt comfortable accessing MySQL data, I attempted to retrieve all the fridge variable's data for a single snapshot. In order to do this I constructed a simple MySQL command within the MySQL database and ran it. The database retrieved all of fridge variables' data for a single fridge timestamp. Once I successfully completed this step I scaled the data retrieval up to try to search for a larger set of data (meaning it spans a certain time frame rather than just one specific moment like before) within MySQL. During this step I constructed the full command I would be using when I pulled MySQL data directly from Matlab. After some trial and error I was able to successfully able to access this larger set of fridge data within MySQL.

The next step was to put together a program in Matlab that would be able to connect to the MySQL database and allow me to directly pull data. This step proved to be tricky because there was a lot of issues that I did not foresee when simply accessing data within MySQL. The first of which was that the sheer amount of data that was being pulled across was causing my program to take multiple hours (data size was about 1 month worth of data). In addition MySQL was a remote database and MySQL calls originating in Matlab were surprisingly slow to execute. In order to solve this issue I decided to have MySQL first average the data in a minute by minute basis and then return the averaged data thus decreasing my amount of data by a factor of ten. This step traded some accuracy for a huge increase in efficiency which was a worthwhile trade-off, especially since the likelihood of the fridge variables values changing significantly within a single minute is unlikely.

The next major issue I ran into while constructing my program was how 
to properly convert the retrieved data into a format MATLAB could read. In order to do this it took a lot of trial and error, but I was able to track down the type of memory allocation each fridge variable should be given. For example the majority of the fridge variables should be stored as a double, which is a common type of memory allocation in Matlab.

The final issue that I ran into was how to align the time of the two sets of data. This was by far the most challenging issue because it had no obvious solution. After some searching I discovered that the SuperCDMS data has a time stamp that was in Unix time. Unix time is a popular way of keeping time in computer programming and its start time is January 1st, 1970. Therefore, I decided the best way to approach this issue was to insert the Unix time stamp into the MySQL data. However this step was not as easy as it sounds. In order to do it I had to give MySQL another command that would return its Unix time and then find a way to place this time stamp in the proper place in the returning data set. This meant editing the original MySQL command so that these objectives could be achieved. Once I was able to solve this issue I needed to test to make sure that the two sets of data were properly aligned. I checked the data alignment in three ways.

1. The first way to check a specific series in MySQL and see if its timestamp was the same as the the new Unix time stamp. The best way to do this was to go back to the MySQL database and enter the fridge data retrieval command from there and use the results to confirm that the two were properly aligned. Once both fridge and detector data were aligned in time, I compared whether or not they were from the same place of time by using the online SuperCDMS fridge data historical plotter. To confirm they were aligned I took the series number for a specific time from both the website and my retrieved data. I then compared whether not they were the same. I found that the two were indeed the same series number so I was able to confirm that the data appeared to be aligned. Therefore I was ready to attempt to run my new program which would pull data back from the MySQL database back to Matlab.

2. The next step was to attempt to run my new program to make sure that it was working correctly. When I ran it the first time I chose a time frame of SuperCDMS testing that would provide a small amount of data (only two days worth of data) and found that all of the data returned correctly. The time alignment was checked in the same was as I have previously mentioned above. In order to confirm the data was aligned this, I looked at one particular variable that contains a well known pattern which can be seen in figure 3.3. When I compared the two I saw that the patterns matched each other closely. Therefore, this was a good sign that that my program was working in a way that was expected.

3. The last way was actually do an initial comparison of the two sets of data to make sure everything was properly aligned. I decided the best way to go about doing this was by plotting the newly-aligned detector data 


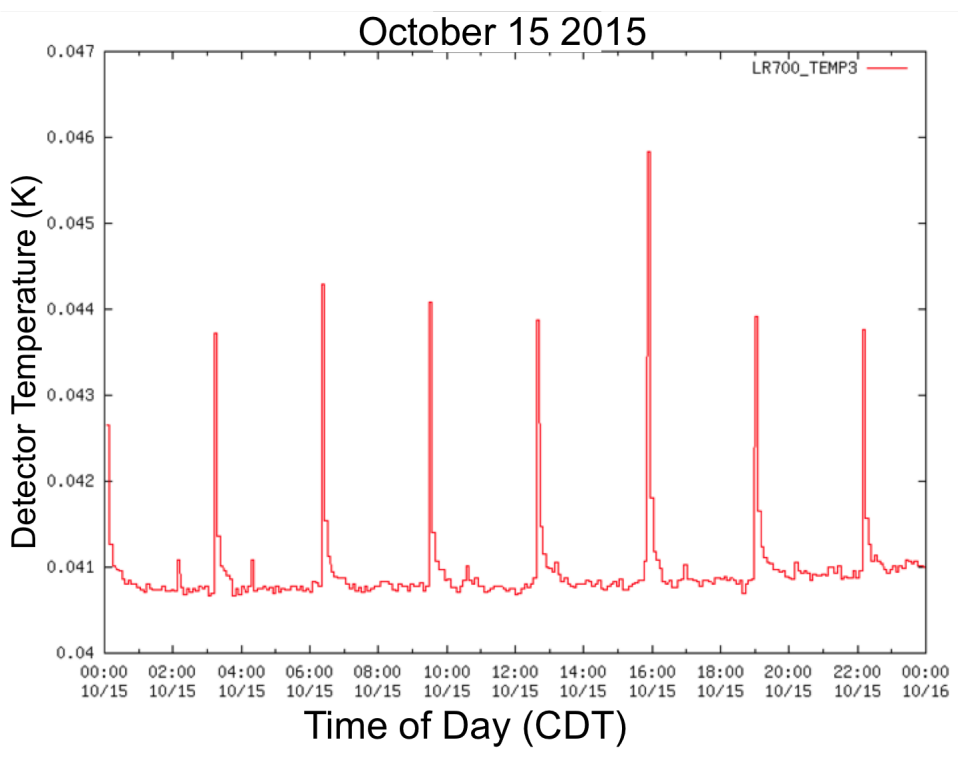

Figure 3.3: This graph is a screen shot of one detector temperature variable from the SuperCDMS fridge. This is important to examine because it contains a well known pattern that could be used to compare with my project's test results. As on can see there are peaks in the temperature every three hours. This is a result from flashing the detectors. Once this is complete the detectors are cooled again to around $40 \mathrm{mK}$. 


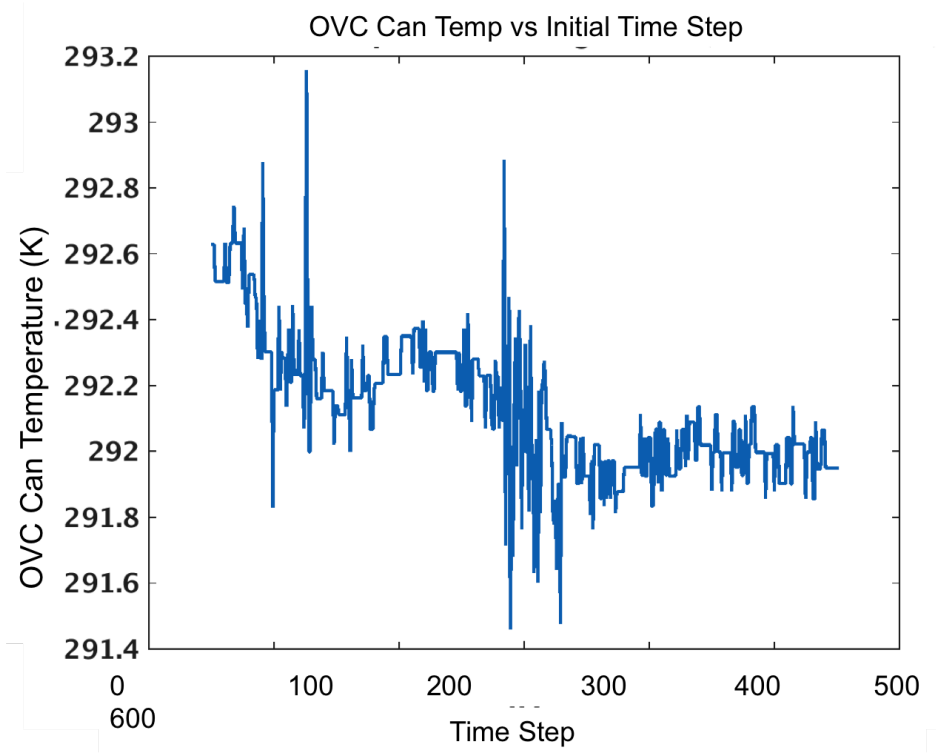

Figure 3.4: This graph shows how the temperature of the Outer Vacuum Can (OVC) varies over time. This plot was used initially to determine if the program was working correctly. When compared with the values from the online SuperCDMS fridge historical plotter it was clear that this program was indeed behaving correctly. This was confirmed by matching series numbers with their given time stamps. If the two series numbers and the time stamps were both the same we knew that the two were properly aligned. 
against one of the returned fridge variables, as can be seen in figure 3.4. This step was designed to make sure all of the data had returned properly. In this particular case I chose the time steps that are given to each piece of data that is taken (this is one of the newly aligned values) on the Outer Vacuum Chamber (OVC) can temperature (this is one of the the 273 fridge variables). By creating this comparison I was able to determine that my program was correctly working and was ready to move on the next step of my project.

\subsubsection{Implementing the Program}

Before I begin I want to briefly describe some important details that will be assumed throughout the rest of this chapter. As I mentioned above, the SuperCDMS fridge contains 273 variables. Each one of these variables performs a specific purpose so there is a possibility that anyone of them could indicate a source of detector noise. However, due to information I was given by a member of the collaboration and through simply viewing the data, many of the variables are highly unlikely to be noise culprits. In addition due to time constraints, through the rest of this chapter we will be focusing on only one variable known as the Outer Vacuum Chamber (OVC) can temperature. I will mention later in detail why this variable was chosen, but simply it is because it provides a set of data that fluctuates a great deal. In addition it is important to note that the SuperCDMS data contains 15 detectors. For the purposes of time we will primarily be looking at detector 1 for all of the tests.

Now that I was able to both obtain and align in time the fridge and detector data, and was able to scale up my fridge retrieval, the next logical step was to begin comparing the fridge data with some of the detector measurements that were taken from SuperCDMS of the detector data. The first detector measurement I decided to try comparing was the charge of the detector (referred to as Volts0). This was a logical choice to pick as my first test because this is a good baseline value within the detector and background noise from the detector is expected to effect this value. Volts0 is a single snapshot of the charge within the detector which gives us clues to the amount of noise within the detector. So the next step was to implement this into my existing data retrieval program by adding a few lines of code that retrieved the Volts0 detector noise data. Once this had been completed, I decided to test to make sure that the data was being retrieved properly. In order to do this I performed a simple data pull (series of 300 data points from run 134) from the SuperCDMS detector data and compared the results with the corresponding fridge data, as can be seen in figure 3.5. This result showed multiple things. First, it showed that my program was behaving in an expected way because all of the points were within in an expected region and the data was the same as the data we saw when just looking at the Volts0 data on its own. Second, it showed was that there may be a potential correlation occurring within this variable because there appears to be two populations being formed. However, it was way to early to make this claim so further testing was required to test whether a correlation between 


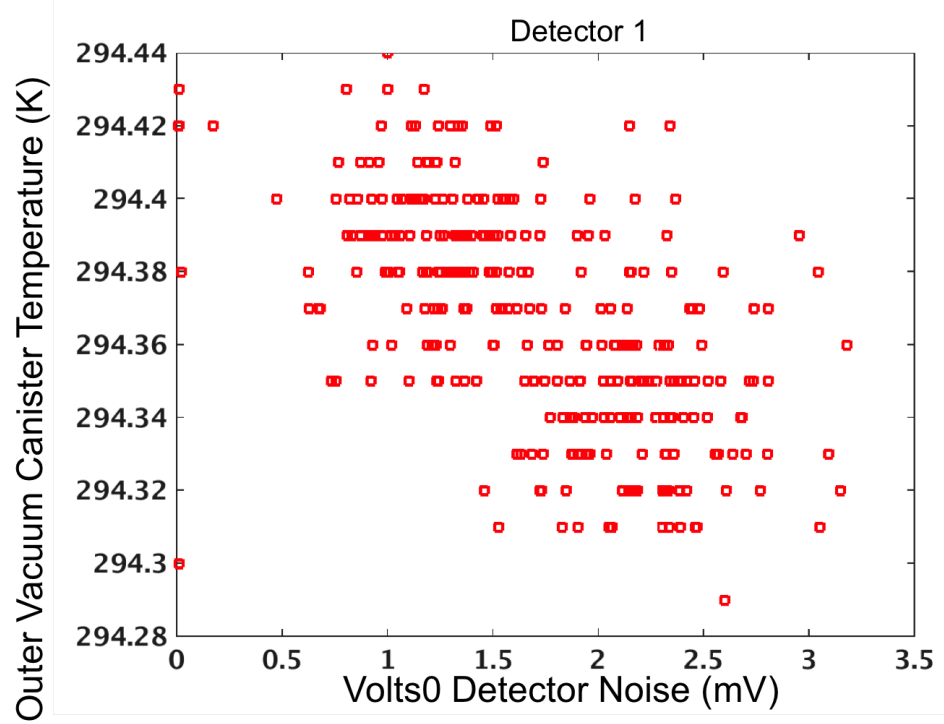

Figure 3.5: Each point on this plot represents a minutes worth of fridge data that has been averaged. SuperCDMS takes a snapshot of their data every 3 seconds which makes this amount that was dealt with in this experiment very large and complex. It is also important to note that it looks like there are two populations or a correlation between the fridge variables. This may be an important result but it could also be coincidental. Therefore, further testing was required to discover if this was anything of value.

detector noise levels and OVC temperature existed.

Once I demonstrated that my program was able to compare the Volts0 to the fridge variables I was ready to start pulling large sets of fridge data. The first larger set of fridge data I pulled was one that lasted nearly 3 weeks. Pulling this data had its own series of issues that needed to be solved. The major issue was how to decrease the amount of time it took for my program to run (referred to as run time). To solve this I decided to remove some of the overlapping steps (in technical terms there were multiple For loops that could be condensed, thus helping reduce the run time. For loops are not very efficient in Matlab so the removal of some of them helped tremendously.) within my program, in an attempt to decrease run time. I was able to successfully reduce my run time from 6 hours down to 4 hours for a set of data that contained 2 months worth of data.

The next step was to refine my data by removing outlier data points. Before I begin, it is important to note that outlying data points existed in both the SuperCDMS detector data and the fridge data. In order to refine my data I attempted to remove all of the outliers from both the SuperCDMS data and 


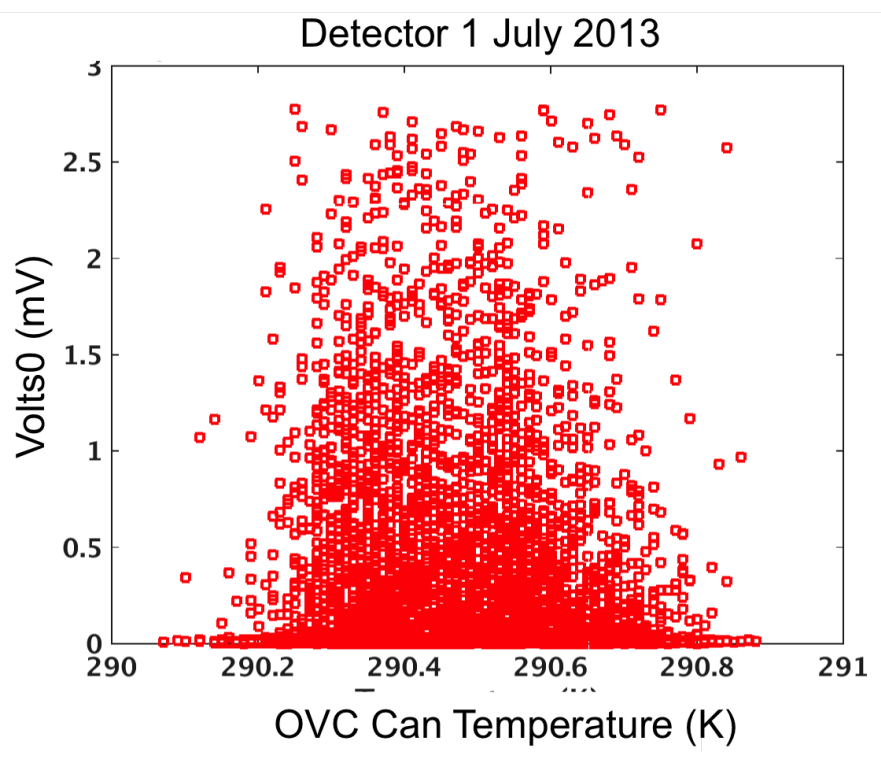

Figure 3.6: A plot that shows the standard deviation of the Ovc can temperature compared to volts. This series of data had all of the outliers removed that were not within the set parameter of $3 \sigma$. This kind of data cut is important to apply because it allows us to examine the bulk of data rather than the outliers. Each point on this plot represents a minutes worth of fridge data that has been averaged.

the fridge data, which can be seen in figure 3.5. These outlying data points are caused by things like actual events occurring within the detector (since we are using a baseline of the noise in the detector, an event will cause a huge spike in voltage) or other outside events occurring. In order to remove them in a an unbiased way I removed all of the data points that were at least 3 standard deviations away $(\sigma)$ away from the mean value. This allowed me to obtain the results shown in figure 3.6. As can be seen, this figure only shows the data for the OVC can temperature. This result allows us to compare the bulk of the data and test for variances within it which could be caused by a potential noise culprit. Therefore this step was one of the most crucial to the project.

At this point, I made a crucial change at this stage in my analysis. Instead of using the Volts0 to view the data from the SuperCDMS detectors, I changed to examining the pre-pulse baseline (PPB) within the detectors. The PPB is a measurement of the the size of the charge when their is no event occurring within the detector, which makes it a much better estimator of the detector noise levels. This was an important change because this measurement allowed me to view a more precise value within the detector whereas Vots0 was just a snapshot view and could be effected by events occurring within the detector. 
When I say general I mean that the PPB gives a measurement that gives a better picture of the normal conditions within the detector. This is important because it potentially helps remove other variables that may falsely show a correlation between noise and the fridge variables. Therefore this switch was an attempt to make my analysis more precise.

Now that I had the tools to determine which fridge variables consistently correlated with an increased level of noise, I was ready to pull large chunks of fridge data and them for evidence of correlated noise. In order to do this I implemented both of the programs I had written and pulled all the fridge data for a three month time span. In order to do this in an efficient way, I pulled small chunks of data at a time (around 3 weeks of data). I then stored each set of data with a unique name that allowed me to recognize what each set of data was. In addition to efficiency, this process also gave more flexibility in the analysis section (which will be discussed in the next section), since multiple time windows within the three month time frame could then be easily compared. Therefore once I was complete with this step I was ready to move onto the analysis step.

\subsubsection{Analysis}

Once my programs were demonstrated to work in a way that was consistent with what was expected and I had pulled a large enough set of data, I was ready to move on to the analysis portion. This step was crucial because it was when I was able to gather results and see if there were any correlations within the variables that would potentially be able to demonstrate that some fridge variables were indicators of detector noise culprits.

Under the time constraints of this thesis, I analyzed my data in two ways:

1. Noise impact across many Detectors- This first way was designed to identify fridge variables that are frequently well correlated with detector noise across many detectors. This was a great way to start because it was a quick and allowed me to identify any potential fridge variables that could be correlated. When a series of data from the SuperCDMS detectors was retrieved we could could give it a correlation coefficient value that was compared to the fridge data. I computed a correlation value for each fridge variable and each detector for a total of 4095 total correlation coefficients. Rather than plot each correlation coefficient, I average them over all 15 detectors and use that average value. The average gives a good overview of the noise impact on the entire experiment. The resulting 276 averaged correlation coefficients could then be compared using a single plot shown in figure 3.7. From looking at this figure we see that the correlation coefficients were small implying that there was little-to-no correlation between detector noise and any of the fridge variables. This was to expected because if the values were high then there would be a major problem with the detectors ability to take sensitive results. However, I was able to look at this plot and see that some variables did seem to have similar, possible significant correlation values in multiple data series. If we look at figure 


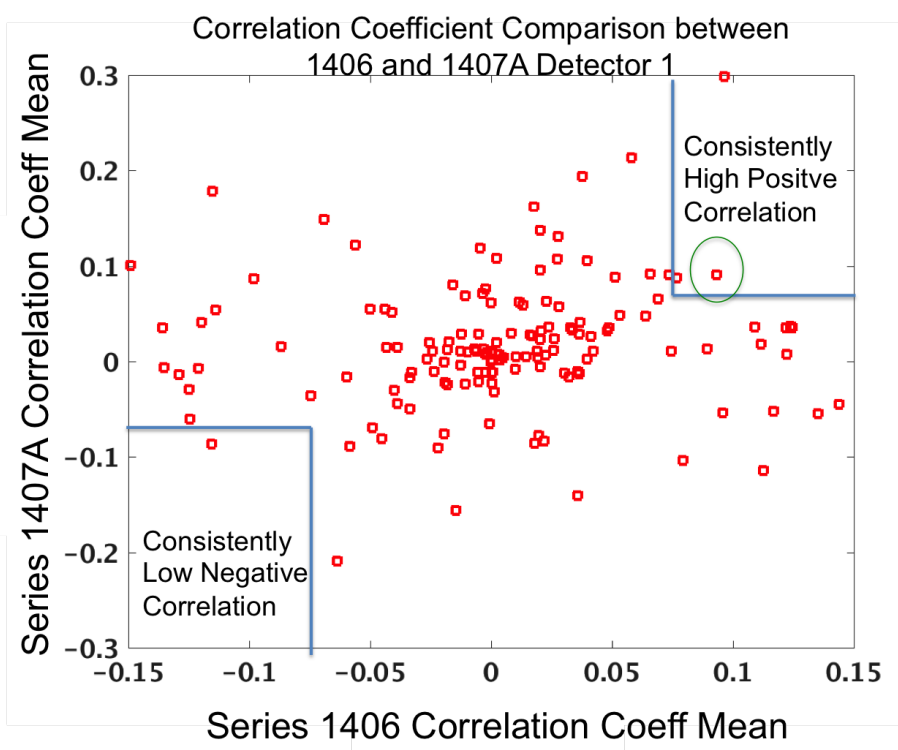

Figure 3.7: This plot shows the average correlation coefficient between detector noise and each of the 273 fridge variables. The $\mathrm{x}$-axis gives the average correlation coefficients for series 1406 . The y-axis gives the average correlation coefficients for series 1407A. Each point represents a different fridge variable's correlation coefficient averaged over all detector noise levels. As on can see the bulk of the averaged correlation coefficients are located near the point of zero correlation $(0,0)$ which is to be expected. However some coefficients have values that may be significantly far from zero. A large correlation coefficient might indicate a possible correlation between a fridge variable and detector noise level. I chose a threshold (|averaged correlation coefficient $\mid>0.75$ ) for identifying coefficients with higher levels of correlation (indicated by the blue boxes) and used these to search for potential culprits. The circled point is the value I . 
3.7 we can see that there is a variable circled in the figure, which may be interesting to look further into because it has a similar correlation value in both sets of data and that value is rather large relative to most of the other values. I identified all the fridge variables that I considered to have a high positive/negative correlation value. This was done by requiring a high correlation levels of .75 in both the $\mathrm{x}$ and $\mathrm{y}$ direction (shown by the boxes in the corner of figure 3.7) that would remain consistent throughout the whole search. If a correlation coefficient fell within this level it was flagged for future study.

Once I had this system in place, it was repeated with each 3 week chunk of data for a 3 month time frame. This 3 month time frame covered 2 full sets (i.e. series 1406 and 1407) of series that were retrieved. When all this was complete I could then look at every recorded variable and see if any of them occurred multiple times. If the variables occurred multiple times I knew this would be a variable worth looking into further because it could potentially be a source of unwanted noise. If a fridge variable wasn't flagged more than 1 time, it was ruled a non-priority candidate but was not entirely ruled out as potential data noise culprit. However, due to time constraints no further research into these values was conducted. Overall, this test was a quick and efficient way to test for any potential noise culprits.I was able to find a few variables, in specific the OVC can temp and Helium in line, that appeared multiple times in the data twice. Therefore, it was important to mark these two variables for further investigation.

2. Testing the noise impact within each detector - The second analysis is designed to test how each detector's correlation coefficient was being effected by each fridge variable. This method was intended to yield a more in depth view of what was going on in a single detector. This was important to analyze because the approach from the above method averaged the detector data rather than looking at each one individually meaning that it may of missed a single detector noise source. An example of this would be if detector 6 had a correlation coefficient that was consistently higher than the rest of the detectors, it may mean some noise source tended to preferentially couple to this detector, but it would of been missed in the first test. To go about completing this method, I wrote a short program to have it store all the relative data i.e. standard deviations and each detectors correlation value. This table gives us a plethora of information that could help target potential noise sources. In particular the table allowed me to examine if a certain variable was causing issues in only one detector. At the time of writing this, no significant results were found.

\subsection{Chapter Summary}

These analyses were useful and is the first attempt at systematically searching all the SuperCDMS fridge variables for correlated detector noise. These analyses 
had many challenges in every step but by breaking it down and taking it step by step I was able to create a set of programs that appear to work correctly. Therefore the next student who picks up from where I left off will have a great start at performing this type of analysis and will be able to continue searching for noise culprits. 


\section{Chapter 4}

\section{Outlook}

The final chapter of this thesis will be a short summary of the outlook on each of these two projects that were performed. We will examine how each of these projects can be taken and built upon by future students into their research projects.

\subsection{Simulation Outlook}

For my simulation project all of the code was already built by SuperCDMS so it is very easy for anyone to pick up from where I left off and expand the tests I performed. The most interesting test that could be expanded on is the impact of the strength of the electric field within the detector on propagation spread size, in particular examining even higher voltages than I chose. This would be interesting to look at because the max voltage I used was only around $100 \mathrm{~V}$ which actually is not all that high for SuperCDMS but is far less than should be possible. It would especially be interesting to simulate at how high the voltage can be turned up within these detectors before things begin to break down within the detector. This could useful because if a tight enough spread could be achieved it could potentially make it much easier to distinguish between a nuclear and electron recoil. Therefore someone in the future may find it interesting to look at voltages higher than this and see how the propagation path of charged particles differ.

There is one key piece of code that I was able to produce for this project that may be interesting for someone in the future to look at. During my experiment I modified a rotational plotting tool in order to work for this type of information. This plotting tool is useful because it allows the user to view the propagation of particles frame by frame in a rotating 3D plot. An example of a few frames produced by this plotting program can be seen in figure 4.1. This tool is very

useful in examining the propagation and may be useful for someone in the future to develop further to aid in understanding future simulations. 


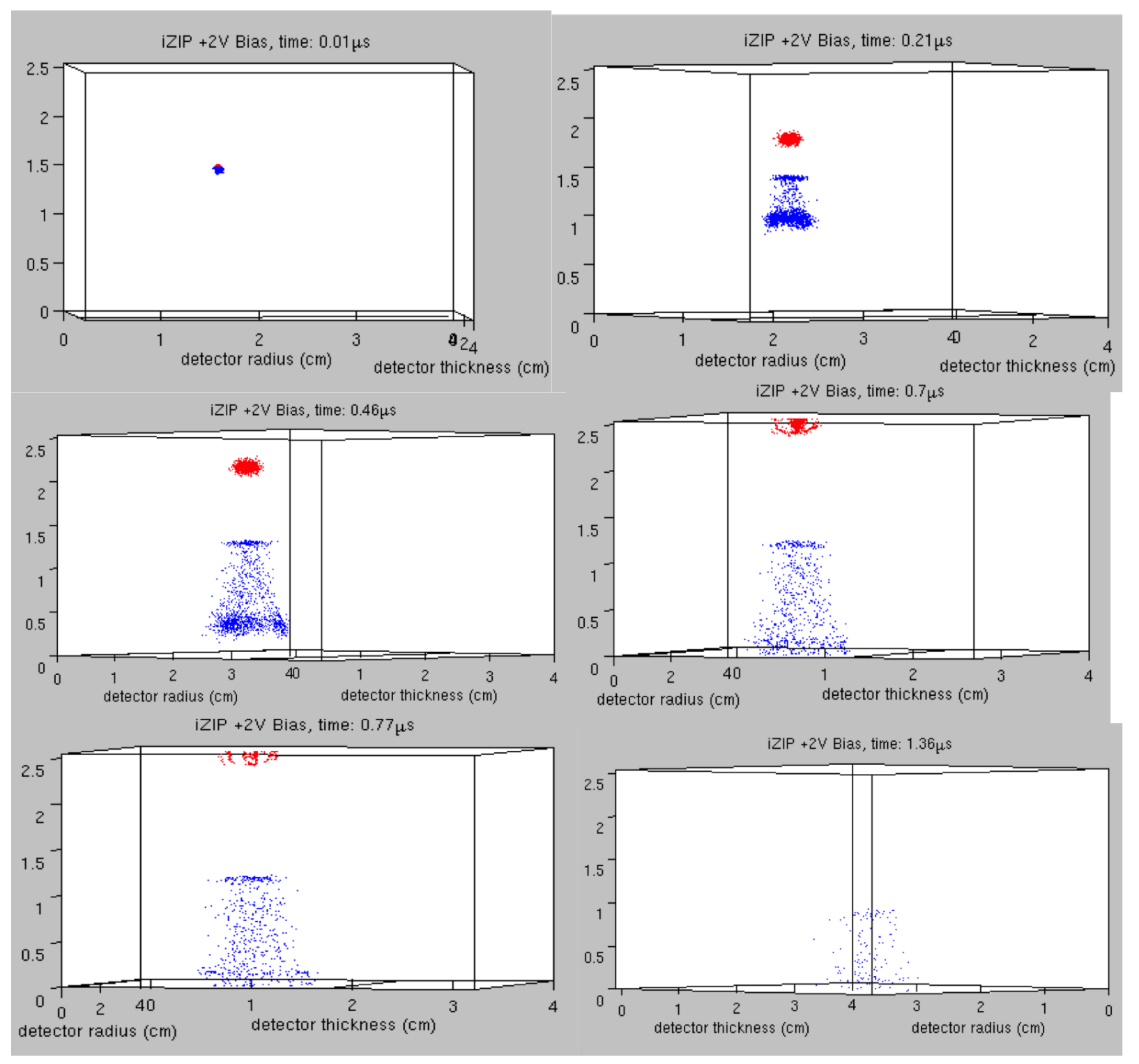

Figure 4.1: This image is a few frames from the rotating plotter that I edited for this simulation. As one can see this can be very useful because the user can view what the propagation looks like at different angles and at different times. As usual the holes are represented by the lighter color traveling up and the electrons are the darker color traveling downwards. The times given in each frame is the time since the particle interaction in the detector. 


\subsection{Analysis Outlook}

For the second project I worked on there is a lot of area for someone in the future to work with going forward. The most obvious way would be by completing the analysis that I had begun on variables from the fridge. The best way to approach this may be by implementing my plotting approach to whatever set of data they choose and start marking which variables seem to have similarly high correlation values. The person could then expand their search through much more data (somewhere in the range of $6-12$ months) and see if the problematic variables remain constant throughout the whole time frame. If they do then it would be interesting to apply my table method and see if these high values are located within just one detector or if they are in all of them. This would help narrow down what is causing the noise and would also help to determine if it is a known issue.

The other way someone could expand this project is by simply using my programs as templates and applying them to other types of analysis projects. My first program is designed to connect to MySQL remotely and then pull data across that remote connection in an efficient way. This part of the program would be useful for anyone to use because it took a lot of trial and error to get it working properly and would be major time saver to simply use my program and edit it for the specific user's needs. The next part of my first program that could be useful is the conversion of the MySQL data into a format that can be read by Matlab. Like before, designing this part of the program was very time consuming so a future user may want to simply take my code and incorporate into their own. In addition, this section of the program could be edited to convert the MySQL into a different language other than Matlab. In order to do this the user would simply need to edit a few lines of the program so that the data would be properly converted into their desired computer language. Therefore this program has many additional uses for projects going forward.

The other program that I wrote that could be useful to future users is my program that creates a mask for the SuperCDMS data. This script has limited abilities outside of this project, but I could see it being taken and used for anyone trying to create a mask for their data. This program is good at dealing with large amounts of data in an efficient way and it would be good teaching mechanism for someone who has never dealt with creating masks.

\subsection{Conclusion}

Over the course of the last two years I have had the honor to work on two extremely interesting projects. These projects have given me an introduction into the world of physics research and have inspired me to continue down this path. These projects characterize two completely different types of research methods but both are integral to learn for any physicist. 


\section{Bibliography}

[1] J. Cartwright. Axions could shed light on solar mysteries. URL http://physicsworld.com/cws/article/news/2009/jun/12/axions-could-shed-light-on-solar-mys

[2] P. T. et al. Astronomy and Astrophys. 2007. URL arXiv:astro$\mathrm{ph} / 0607207$.

[3] S. L. et al. Monte Carlo Comparisons to a Cryogenic Dark Matter Search Detector with low Transition-Edge-Sensor Transition Temperature. 2001. URL http://arxiv.org/pdf/1109.1198v1.pdf.

[4] S. Fallows. Measurement of Nuclear Recoils in the CDMS II Dark Matter Search. URL http://cdms. berkeley.edu/Dissertations/fallows.pdf.

[5] NASA. Dark energy, dark matter. URL http://science.nasa.gov/astrophysics/focus-areas/what-is-dark-energy/.

[6] R. Pecci. The Strong CP Problem and Axions. Lecture Notes in Physics, 24 Jul 2006. URL http://arxiv.org/pdf/hep-ph/0607268v1.pdf.

[7] S. Soter and N. deGrasse Tyson. COSMIC HORIZONS: ASTRONOMY AT THE CUTTING EDGE. New Press, 2000.

[8] SuperCDMS. SuperCDMS detectors. URL http://cdms. berkeley.edu/experiment.html. 